\title{
The influence of numerical parameters on tidally triggered bar formation
}

\author{
R. F. Gabbasov ${ }^{1,2}$, M. A. Rodríguez-Meza ${ }^{1}$, J. Klapp ${ }^{1,3}$, and J. L. Cervantes-Cota ${ }^{1}$ \\ 1 Depto. de Física, Instituto Nacional de Investigaciones Nucleares, Apdo. Postal 18-1027, México DF 11801, México \\ e-mail: ruslan@nuclear.inin.mx \\ 2 Facultad de Ciencias, Universidad Autónoma del Estado de México, Instituto Literario 100, CP 50000, Toluca, México \\ 3 Universität Konstanz, Fachbereich Physik, Fach M568, 78457 Konstanz, Germany
}

Received 24 September 2005 / Accepted 19 December 2005

ABSTRACT

The joint influence of numerical parameters such as the number of particles $N$, the gravitational softening length $\varepsilon$ and the time-step $\Delta t$ is investigated in the context of galaxy simulations. For isolated galaxy models we have performed a convergence study and estimated the numerical parameters ranges for which the relaxed models do not deviate significantly from its initial configuration. By fixing $N$, we calculate the range of the mean interparticle separation $\lambda(r)$ along the disc radius. Uniformly spaced values of $\lambda$ are used as $\varepsilon$ in numerical tests of disc heating. We have found that in the simulations with $N=1310720$ particles $\lambda$ varies by a factor of 6 , and the corresponding final Toomre's parameters $Q$ change by only about 5 per cent. By decreasing $N$, the $\lambda$ and $Q$ ranges broaden. Large $\varepsilon$ and small $N$ cause an earlier bar formation. In addition, the numerical experiments indicate, that for a given set of parameters the disc heating is smaller with the Plummer softening than with the spline softening. For galaxy collision models we have studied the influence of the selected numerical parameters on the formation of tidally triggered bars in galactic discs and their properties, such as their dimensions, shape, amplitude and rotational velocity. Numerical simulations indicate that the properties of the formed bars strongly depend upon the selection of $N$ and $\varepsilon$. Large values of the gravitational softening parameter and a small number of particles result in the rapid formation of a well defined, slowly rotating bar. On the other hand, small values of $\varepsilon$ produce a small, rapidly rotating disc with tightly wound spiral arms, and subsequently a weak bar emerges. We have found that by increasing $N$, the bar properties converge and the effect of the softening parameter diminishes. Finally, in some cases short spiral arms are observed at the ends of the bar that change periodically from trailing to leading and vice-versa - the wiggle.

Key words. galaxies: kinematics and dynamics - galaxies: interaction - methods: $N$-body simulation

\section{Introduction}

One of the long-standing issues in $\mathrm{N}$-body simulations is how a specific selection of numerical parameters influence an outcome. In astrophysical and cosmological simulations there are a number of these parameters that may play an important role, and therefore they have to be carefully selected. For instance, it is well known that collisionless simulations of galaxies are performed with a number of particles $N$, that is far less than the number of stars in a typical galaxy, hence, it induces artificial non-uniformities of the gravitational field. In order to smooth the field and to avoid the divergence of the gravitational force when the separation between particles becomes small, close encounters between particles should be avoided. This is usually done by softening the force between two particles using some modified form of the Newtonian gravitational potential. For example, the Plummer softening is widely used since the first simulations of Aarseth (1963). Other softening algorithms exist that give a better approximation to the Newtonian force such as, for example, higher powers of the Plummer softening
(Athanassoula et al. 2000) or the spline softening (Hernquist \& Katz 1989), among others (Dehnen 2001). The degree of the force softening is defined by the gravitational softening parameter $\varepsilon$. Small values of $\varepsilon$ cause high relaxation rates but give a better resolution of the internal structure of the system. On the other hand, a large softening reduces the relaxation rate, but errors in the force calculation increase because of the deviation from the Newtonian force.

The influence of $\varepsilon$ on the force calculation error was discussed by Merrit (1996), where he found that its optimal choice is related to the minimum error that is characterized by the so-called bias and variance. He also gives two empirical expressions to estimate $\varepsilon$ for the Plummer (1911) and Hernquist (1990) density profiles. This work was later extended to another configurations (Athanassoula et al. 1998, 2000) and to other softening kernels (Dehnen 2001). Although these criteria give $\varepsilon$ that minimizes the force errors, they have been derived for rigid Monte-Carlo realizations, each of which, when evolved in time, will drift to its individual equilibrium state. Such criteria allow to estimate only the magnitude of $\varepsilon$ that minimizes 
the initial perturbation due to the modification of gravity, but ignores the further evolution. For this reason it is important to check whether these criteria apply for dynamical evolving systems such as galaxies.

Two-body relaxation effects and force calculation errors depend on $\varepsilon$ and $N$, and both of them should be simultaneously minimized. It is, however, difficult to measure them separately (Hernquist 1993b), e.g. they manifest themselves as a joint error in the energy of the particles (Hernquist \& Barnes 1990).

Another source of error stems from an inadequate choosing of the integration time-step $\Delta t$, which also results in poor energy conservation. This latter error is typically influenced by the maximum acceleration, which is constrained by the softening parameter. In addition, there are truncation and roundoff errors, but they are small and can be easily controlled.

The problem of an adequate selection of numerical parameters $(N, \varepsilon, \Delta t)$ for $N$-body simulations has been widely discussed in the literature, usually separately. In particular, concerning the effect of the softening parameter in 2D galaxy simulations, it was established that in addition to reduction of relaxation, it plays a stabilizing role, and the meaning of a given value of the $Q$ parameter strongly depends on the value of $\varepsilon$ (Miller 1971; Romeo 1994). Indeed, relaxation can heavily affect the results of numerical studies of disc stability (e.g, White 1988). Criteria for choosing $\varepsilon$ for such systems, based on dynamical requirements and type of softening, were discussed by Romeo $(1994,1997,1998)$. A 3D study to estimate the numerical parameters was made by Hernquist $(1987,1993 b)$ in which the influence of the softening parameter and the number of particles on the force errors were investigated. Also, there is a rich discussion concerning the choosing of numerical parameters and its effects on cosmological simulations and small scale structure formation (e.g., Power et al. 2003). On the other hand, analytical estimations give severe limits on the reliability of $N$-body calculations. For instance, it was shown that the orbits of particles diverge exponentially with time from their original trajectories (Goodman et al. 1993; Hayes 2003).

For galaxy simulations, the above mentioned effects influence any dynamic process, such as the formation of bars in spirals that we study in the present work. This problem involves physical as well as numerical aspects. Since the 70s, several numerical simulations have analysed this problem and found that cold, rotationally supported stellar discs are globally unstable to bisymmetric distortions and quickly evolve into well defined spontaneous bars (e.g., Hohl 1971). The formation of a bar in unstable disc galaxies was extensively studied in both two- and three-dimensional simulations (e.g., Sellwood 1981; Sellwood \& Carlberg 1984; Sellwood \& Athanassoula 1986; Athanassoula \& Sellwood 1986). It was established that the bar formation can be suppressed by introducing high enough velocity dispersions (Toomre 1964), or by a massive spherical halo (Ostriker \& Peebles 1973). The 2D-simulations have shown that the bar's length is not only defined by physical parameters but also strongly depends on the magnitude of the softening parameter (Sellwood 1981; Sellwood \& Carlberg 1984). Furthermore, recent 3D-simulations of isolated galaxies (Debattista \& Sellwood 1998, 2000; Athanassoula \& Misiriotis 2002) have shown that the bar semi-major axis is more than twice as long as the observed length, which is close to the exponential length-scale of the disc (Elmegreen \& Elmegreen 1985). On the other hand, it was found that bar rotation velocities were slowed down to less than twice the observed velocities in just a few Gyrs (Debattista \& Sellwood 2000; Athanassoula $\&$ Misiriotis 2002). This result coincides with predictions of Weinberg (1985) that bars are slowed down by dynamical friction with a massive halo. However, it was argued that this slowdown is partially associated with the low halo spatial resolution that diminishes the rotational velocity of the formed bar (Valenzuela \& Klypin 2003). A high resolution simulation with 20 million particles (O'Neill \& Dubinski 2003) still indicates the bar slowdown, although it is not so drastic. Moreover, it was found that even in a stable galactic disc a bar may emerge due to discreteness of the halo (Walker, Mihos \& Hernquist 1996), and Athanassoula $(2002,2003)$ showed that these effects are related to the disc's angular momentum redistribution and resonances of halo particles.

Numerical experiments performed by other authors have shown that bars may also form in disc galaxies which interact with a companion galaxy (Nogushi 1987; Gerin et al. 1990; Salo 1990). A systematic 2D study of tidally triggered bar formation (Salo 1990) in spiral galaxies has shown that bar formation depends simultaneously on the shape of the rotational curve, the disc-to-halo mass ratio, the halo concentration, the strength and the geometry of the perturbation. These results have been partially confirmed by self-consistent 3D simulations (Barnes 1992; Miwa \& Nogushi 1998; Berentzen et al. 2004), but the formed bars are found to be slower than in isolated models, indicating a possible explanation for the dichotomy of bar characteristics found by Elmegreen \& Elmegreen (1985).

Motivated by the above arguments, in this work we investigate for an isolated galaxy in equilibrium and for the collision of two galaxies, the range of the numerical parameters $(N, \varepsilon$, $\Delta t$ ) that permit us to minimize numerical artifacts in simulations. In addition, we compare the spline gravitational softening with the Plummer one. For the collision of two disc galaxies we study the influence of the numerical parameters on the formation and characteristics of tidal bars.

This work is organized as follows: in Sect. 2 we describe the initial conditions of the galaxy model and the way in which the numerical parameters are computed. We obtain a particular range for $(N, \varepsilon, \Delta t)$, which in Sect. 3 is tested to observe deviations from an initial configuration. Based on a convergence study we choose the most suitable values for the numerical parameters. Then, in Sect. 4 we analyse the influence of the parameters on the bar formation after the collision of two spirals. Finally, in Sect. 5 we discuss the results and in Sect. 6 outline our conclusions.

\section{Numerical modelling}

A self-consistent galaxy model is usually constructed with particles that are governed by the collisionless Boltzmann equation coupled to the Poisson equation for the Newtonian gravitational potential. As mentioned above, the gravitational potential is softened and, ideally, one should construct a 
self-consistent model for the potential. For simplicity, a standard practice is to use an algorithm based on the exact Newtonian potential, but this requires to relax the initial galaxy for several crossing times before it can be used for actual simulations. Using this prescription, we follow Barnes (1998) for the construction of the initial conditions. In this model the bulge and the halo are both non-rotating spherically symmetric systems with isotropic velocity dispersions.

The bulge density profile is given by (Hernquist 1990):

$\rho_{\mathrm{b}}(r)=\frac{M_{\mathrm{b}} a_{\mathrm{b}}}{2 \pi} \frac{1}{r\left(r+a_{\mathrm{b}}\right)^{3}}$,

and for the halo we use Dehnen's $\gamma=0$ density profile (Dehnen 1993):

$\rho_{\mathrm{h}}(r)=\frac{3 M_{\mathrm{h}}}{4 \pi} \frac{a_{h}}{\left(r+a_{\mathrm{h}}\right)^{4}}$.

Despite the high concentration of this halo model, it was widely used by Barnes in his galaxy collision simulations. The disc profile is assumed exponential (Freeman 1970):

$\rho_{\mathrm{d}}(r, z)=\frac{M_{\mathrm{d}} \alpha^{2}}{4 \pi z_{0}} \mathrm{e}^{-\alpha r} \operatorname{sech}^{2}\left(\frac{z}{z_{0}}\right)$.

In Eqs. (1)-(3) $M_{\mathrm{b}}, a_{\mathrm{b}}$ and $M_{\mathrm{h}}, a_{\mathrm{h}}$ are the mass and scale-length of the bulge and the halo, respectively, and $M_{\mathrm{d}}, \alpha^{-1}$ and $z_{0}$ are the mass, the scale-length and the scale-height of the disc, respectively.

Using the Monte-Carlo technique, the density profiles are represented by a system of $N$ equal mass bodies, $N$ being the total number of particles. For reducing two-body relaxation effects and to avoid the mass segregation (e.g., Farouki $\&$ Salpeter 1994) we take equal mass particles. The number of particles in each component is assigned in proportion to its mass: $M_{\mathrm{b}}: M_{\mathrm{d}}: M_{\mathrm{h}}=1: 3: 16$. For each component, the mass distribution is truncated at a radius that contains 95 per cent of its total mass, that would correspond to an infinite radius.

The disc vertical dispersion $\sigma_{z}^{2}(r)$ is found from an isothermal sheet equilibrium condition, and the radial velocity dispersion is $\sigma_{r}^{2}(r)=4 \sigma_{z}^{2}(r)$. The azimuthal velocity dispersion is calculated using the epicyclic approximation $\sigma_{\phi}^{2}(r)=$ $\sigma_{r}^{2}(r) \kappa^{2} / 4 \Omega^{2}$, where $\kappa$ is the epicyclic frequency and $\Omega$ the angular velocity. The net rotational velocity of the disc is computed from the asymmetric drift equation (Binney \& Tremaine 1987). The bulge and halo dispersion velocities are calculated using Eq. (14), see below. This equation was integrated numerically, including the masses of all components. Then, isotropic velocities are drawn from the Gaussian distribution. For a detailed description of the initial conditions see Barnes (1998) and Hernquist (1993a). The parameters for our galaxy model are summarized in Table 1, where the columns from left to right give the mass, the number of particles, the cut-off radius and the length scale of each component. We use Barnes's model parameters and system of units. The disc's scale-height is $z_{0}=0.007$. The mass, length and time scales are set to $2.2 \times 10^{11} M_{\odot}=1$, $40 \mathrm{kpc}=1$ and $250 \mathrm{Myr}=1$. In these units, the gravitational constant is $G=1$. The half mass radius of the galaxy is located at $R_{1 / 2} \approx 11 \mathrm{kpc}$. The total rotational curve and rotation curves of each component are shown in Fig. 1.
Table 1. Parameters of the galaxy model. The units of mass and length are $2.2 \times 10^{11} M_{\odot}$ and $40 \mathrm{kpc}$, respectively.

\begin{tabular}{llrcr}
\hline \hline Component & Mass & $\begin{array}{r}\text { Number of } \\
\text { particles }\end{array}$ & $\begin{array}{r}\text { Cutoff } \\
\text { radius }\end{array}$ & $\begin{array}{r}\text { Scale- } \\
\text { length }\end{array}$ \\
\hline Bulge & 0.0625 & $0.05 N$ & 1.5 & 0.04168 \\
Disc & 0.1875 & $0.15 N$ & 0.4 & 0.0833 \\
Halo & 1.0 & $0.8 N$ & 6.0 & 0.1 \\
\hline
\end{tabular}

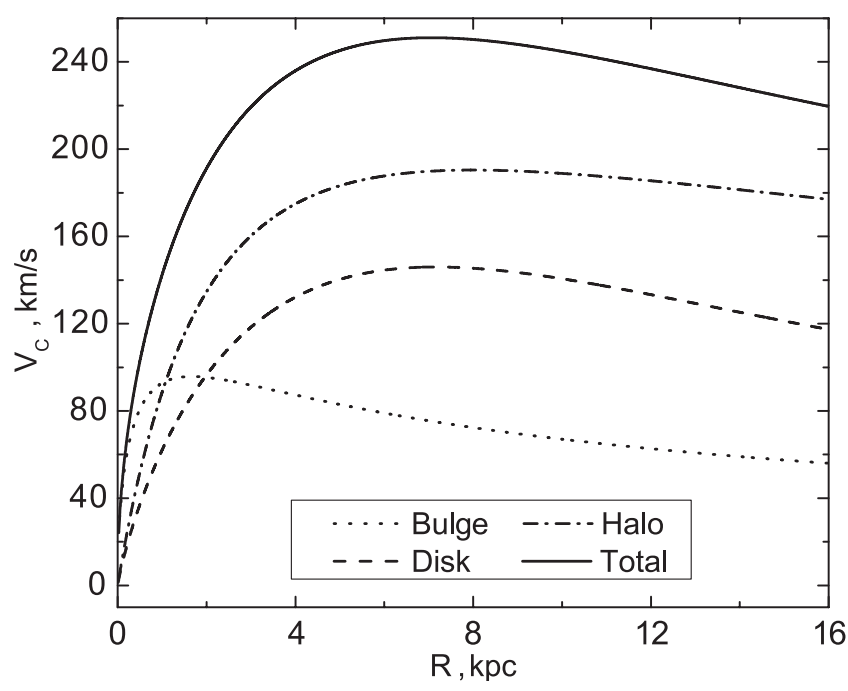

Fig. 1. Rotation curves of the model. The bulge and halo rotation curves are shown up to the radius of the disc.

\subsection{Methods}

For the time evolution we use a hierarchical General Body Smoothed Particle Hydrodynamics (GBSPH) treecode written by one of us (MARM, see details in www.astro.inin. $\mathrm{mx} / \mathrm{mar} /$ nagbody) which is similar to Barnes' treecode (Barnes \& Hut 1986). We use the Plummer softened point-mass potential, and the forces are computed up to quadruple terms with a tolerance parameter $\theta=0.75$. The equations of motion are integrated using a second order leap-frog algorithm with a fixed time-step. The SPH part was switched off.

To verify our results, we also performed several runs using the serial and parallel versions of the public GADGET code (Springel et al. 2001). The main difference between the codes is that for the Newtonian potential, GADGET uses a spline approximation. The latter calculates for the given Plummer softening the equivalent spline softening $h=2.8 \varepsilon$. In order to maintain numerical similarity, we have fixed the time-step to a single value and set the rest of the parameters equal to those used by the GBSPH code. Also, the tree structure was updated at each time-step.

\subsection{Selection of the numerical parameters}

For numerical studies one pursues to have a collisionless, stable equilibrium system. But, due to discreteness effects and force errors this condition is not perfectly fulfilled. If the numerical 
parameters are accordingly chosen, a system that closely matches an initial stable equilibrium during a specific time can be achieved. Thus, the selection of the parameters is of primary importance in any $N$-body simulation.

In spite of the many works on the subject there is still no suitable criterion to define the appropriate magnitude of $\varepsilon$ for a given number of particles. Usually, $\varepsilon$ is chosen in an ad hoc way, or by performing various runs with different $\varepsilon$ and selecting the one that gives a better conservation of the total energy and angular momentum and/or an initial density profile. But this procedure demands a large amount of computational time and is not always performed. On the other hand, a common practice to verify the results is to rerun the simulation with higher $N$, leaving $\varepsilon$ and $\Delta t$ unchanged.

In fact, given the density profile and the number of particles one can roughly estimate the required values of the gravitational softening parameter and the time-step. We now proceed to analyse the criteria for choosing the numerical parameters in order to constrain their possible values for the above galaxy model. This subsection has a preparatory purpose for the numerical simulations of the next section, that should further confine the parameters. The set of parameters that minimize the disc heating and total energy errors will be called "optimal".

\subsubsection{The number of particles}

It is well known that in collisionless $N$-body simulations, the number of particles should be as large as possible. With the new parallel codes it is now possible to simulate galactic systems with $N \gtrsim 10^{6}-10^{7}$.

The minimum number of particles $N$ required to sample a mass $M$ inside an homogeneous sphere of radius $R$ can be estimated from the relaxation time for the softened potential (Huang et al. 1993; Athanassoula et al. 2001):

$\frac{t_{\text {relax }}}{t_{\text {cross }}}=\frac{N}{8 \ln (R / \varepsilon)}$,

where $t_{\text {cross }} \simeq(G \bar{\rho})^{-1 / 2}$ is the crossing time, and $\bar{\rho}$ is the mean density of the system. Once $\varepsilon$ is defined and the $t_{\text {relax }}$ is demanded to be comparable to the age of the universe, the value of $N$ can be found. For example, by requiring $t_{\text {relax }}>$ $10^{10}$ years, the halo model given by Eq. (2) with $t_{\text {cross }} \approx$ $1.5 \times 10^{9}$ years and $\varepsilon=0.01$ will need a minimum $N_{\min } \approx 3300$, which is quite a small number. However, numerical simulations (Athanassoula et al. 2001) show that this condition underestimates the value of the $N$ for centrally concentrated systems by roughly an order of magnitude.

On the other hand, for realistic galactic disc simulations, the vertical structure should be resolved, although the general structure in the plane of the disc can be simulated even when $\varepsilon>z_{0}$ (Zotov \& Morozov 1987). In order to resolve the vertical structure of the disc component given by Eq. (3), one may require $\lambda\left(R_{1 / 2}\right) \leq z_{0}$, where $\lambda$ is the mean interparticle separation estimated within the disc's half-mass radius $R_{1 / 2}$, and $z_{0}$ is the vertical scale height (Hernquist 1987). For our model this gives $N_{\min } \approx 10^{4}$ disc particles. In principle, this number can be considered as an acceptable minimum for our galaxy model.
However, in order to make a more realistic estimation we have to further analyse the dependence of $N$ on $\varepsilon$ and $\Delta t$.

\subsubsection{The softening parameter}

For a given minimum relaxation time and $N$, the magnitude of $\varepsilon$ depends on the mass distribution of the gravitating system. In a series of papers Romeo (1998, and references therein) has analysed the dispersion relation for a $2 \mathrm{D}$ disc and discussed the criteria for physically consistent values of the softening parameter. It was found that stability and relaxation impose different requirements on $\varepsilon$. Although this fact also have implications for $3 \mathrm{D}$ discs, the question in this case is much more complicated because the particles' vertical motion has to be taken into account.

For an homogeneous 3D mass distribution a natural choice for $\varepsilon$ is to be equal to the mean interparticle separation $\lambda$. But astrophysical systems are centrally condensed and the mean interparticle separation is determined by local averages, hence $\varepsilon$ is a local parameter. In practice, however, some authors prefer to compute the minimum constant $\varepsilon$ that optimize some force accuracy level (Hernquist 1987). An inconvenience arises for very inhomogeneous systems since the election is not obvious.

There are several popular criteria that are widely used:

1. Zotov \& Morozov (1987) have suggested that the softening parameter of a gravitating disc can be taken proportional to the radius of interaction, $r_{\text {int }}$, of the particles in its plane, which is given by

$r_{\text {int }}^{2}=\frac{m}{M(r)} r^{2}$,

where $m$ is the mass of an individual particle and $M(r)$ is the total mass of the disc inside the radius $r$. The field is considered smooth if the distance at which the interaction force between a test particle and its nearest neighbour is equal to the force between the same particle and the rest of the particles in the disc. The system is said to be collisionless if the softening parameter is chosen such that $\varepsilon>r_{\text {int }}$.

2. Hernquist (1987) have studied force calculation errors, produced by small number of particles and the Plummer softening of the gravitational potential. He showed that errors decrease weakly with increasing $N$, and are minimized if $\varepsilon<\lambda$, where $\lambda$ is the mean interparticle separation evaluated within the half-mass radius $R_{1 / 2}$. For $\varepsilon>\lambda$ the errors increase rapidly. The latter is explained, particularly for treecodes, by the fact that the expansion of cluster potentials is accurate only for pointlike particles, and it fails for highly softened particles (Hernquist 1987). Then, Newton's third law is violated and linear momentum is badly conserved. Thus, this criterion can be used to impose an upper limit for $\varepsilon$.

3. For a spherical gravitating system the value of $\varepsilon$ that minimizes force errors can be estimated from an empirical relation (Merrit 1996):

$\varepsilon_{\mathrm{a}}=A N^{a}$,

where $A$ and $a$ are parameters that depend on particular models. For example, for a Dehnen's $\gamma=0$ density profile 
of total mass $M=1.0$, scale-length $a=0.1$ and the cutoff radius $R=299.8$, their values are $A=0.12$ and $a=-0.27$, respectively (Athanassoula et al. 2000). However, as it was mentioned in the introduction, this criterion only reduces the initial shot noise, but does not guarantee that the softening chosen in such way will be valid for a long term evolution.

Thus, combining these criteria, it is possible to restrict the values of the softening for a gravitating system. Let us consider a spherical gravitating system of mass $M$ in steady state equilibrium. It can be divided in spherical shells of thickness $\Delta r$ whose volumes are

$\Delta V(r, r+\Delta r)=\int_{r}^{r+\Delta r} \mathrm{~d}^{3} \boldsymbol{r}$.

Given the density profile $\rho(\boldsymbol{r})$, the mass of the shell is

$\Delta M(r, r+\Delta r)=\int_{r}^{r+\Delta r} \rho(\boldsymbol{r}) \mathrm{d}^{3} \boldsymbol{r}$.

If the system is represented by a Monte Carlo distribution of $N$ particles with equal masses $m=M / N$, then the number of particles in that shell is roughly

$\Delta N(r, r+\Delta r)=\frac{\Delta M(r, r+\Delta r)}{M} N$.

The mean interparticle separation in the shell is then given by

$\lambda(r, r+\Delta r)=\left[\frac{\Delta V(r, r+\Delta r)}{\Delta N(r, r+\Delta r)}\right]^{1 / 3}$.

Thus, for each shell a corresponding softening $\varepsilon(r) \propto \lambda(r)$ can be found. However, one cannot use a radially varying gravitational softening in the simulations, since it will lead to energy non-conservation (Zotov \& Morozov 1987). To avoid this, one needs a single value of $\varepsilon$ to be used as the gravitational softening parameter for the whole system. When a value of $\varepsilon$ is estimated at some radius $R_{\text {res }}$, it defines the radius of the system within which the structure is poorly resolved and the obtained results for this region have to be treated with caution. Figure 2 shows $\lambda(r)$ for the above Dehnen's sphere represented by $N=262144$ particles together with the values of (a) $\varepsilon_{\mathrm{a}} \approx 0.0041$ and (b) $\lambda\left(<R_{1 / 2}\right) \approx 0.0122$, that are marked by arrows. Alternatively, one may use the interaction radius defined by Eq. (5) as an estimate for $\varepsilon$. The interaction radius $r_{\text {int }}$, calculated assuming circular orbits, increases linearly with radius from zero to roughly 3.31 at the cutoff radius. Using these criteria the possible values of softening are confined inside the striped area.

In order to estimate the inferior limit for $\varepsilon$, the following procedure can be used. The Monte-Carlo particles are indeed agglomerations of $\sim 10^{4}-10^{6} M_{\odot}$ when compared to real galaxies. Assuming the particles to be Plummer's spheres with masses $m$ and half-mass radii $r_{\mathrm{h}}$, they can be treated as point masses if the local mean separation $\lambda \geq 2 r_{\mathrm{h}}$. In order to estimate the value of $\varepsilon$, we can use the same opening criterion $\theta$

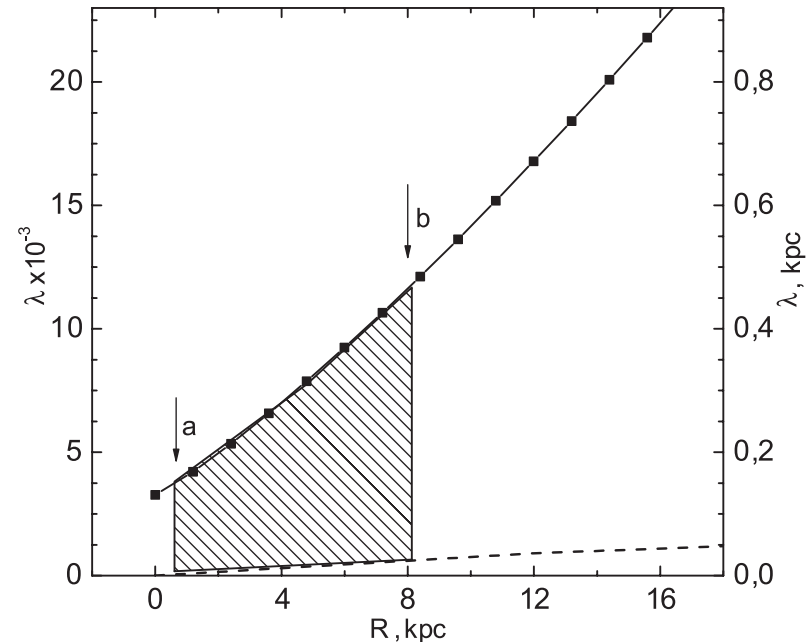

Fig. 2. Mean interparticle separation as function of radius for Dehnen's $\gamma=0$ density profile (upper curve), and the interaction radius (lower, dashed curve). The curves are plotted only up to $R=$ $18 \mathrm{kpc}$ in order to show the behavior near the centre. The arrows mark (a) the value of $\varepsilon_{\mathrm{a}}$ calculated from Eq. (6), and (b) $\lambda$ evaluated within the half-mass radius.

that is used in treecodes. The structure of the mass distribution is unresolved when $2 r_{\mathrm{h}} / \lambda \leq \theta$. The softening radius of a Plummer's sphere is related to $r_{\mathrm{h}}$ as $1.3 \varepsilon \approx r_{\mathrm{h}}$, and thus

$\varepsilon=\theta \lambda / 2.6$.

If $\lambda$ is known, a rough value of $\varepsilon$ can be calculated. As shown by Hernquist (1987), for treecodes the value of $\theta \in$ (0.7-1) represents a compromise between accuracy and performance. However, there is an inconsistency in implementing the Plummer softening due to force calculation as it would be between a point mass and a Plummer sphere, instead of between two point masses (Dyer \& Ip 1993). With the above assumption, the latter is weakened, but not completely removed because of the infinite extension of the Plummer distribution. While this inconsistency should be taken into account in simulations of systems such as globular clusters, some authors argue it is irrelevant for galaxy simulations (e.g. Romeo 1998; Dehnen 2001). On the other hand, the study of Hayes (2003) shows that the adequate softening is in the range $\varepsilon \in$ [0.2-1] times the mean interparticle separation, in agreement with Eq. (11) and Fig. 2.

For each component (bulge, disc and halo) of the galaxy model, the mean interparticle separation can be easily evaluated as a function of radius using Eqs. (7)-(10), and $\varepsilon$ from Eq. (11). Once $\varepsilon$ is defined for each component, it can be used as an input softening parameter for codes such as GADGET that handle individual $\varepsilon$ for each component. Individual gravitational softenings are preferable in simulations, but the total energy is less conserved and Newton's third law is strongly violated (Dyer \& Ip 1993). To avoid this problem individual softenings should be somehow symmetrized in order that particles have the same maximum force. Besides, the $\varepsilon$ found in this way does not include the mass distribution of the other components.

If a single value of $\varepsilon$ is used for a whole galaxy model, then the question becomes more complicated, and some averaged 


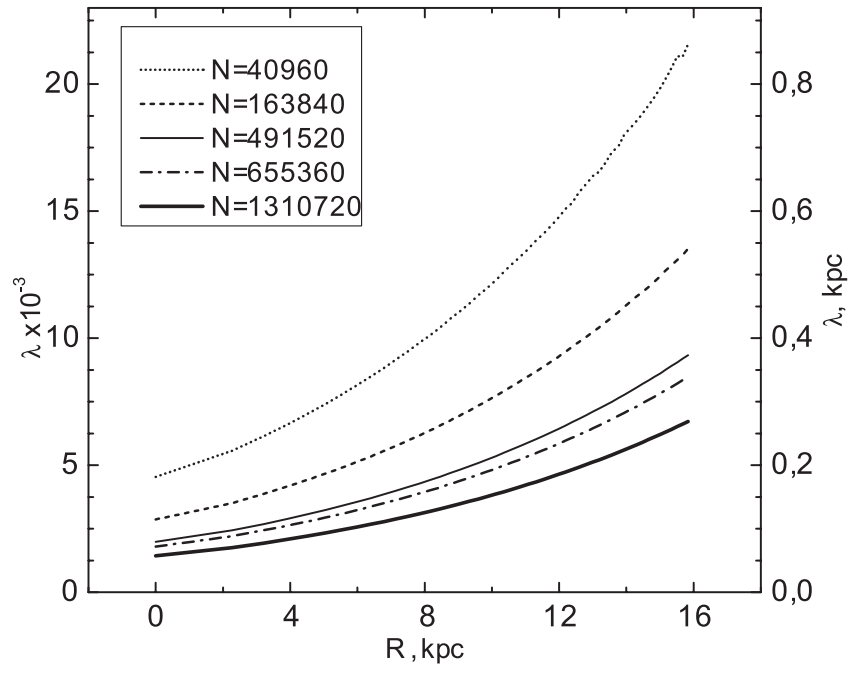

Fig. 3. Mean interparticle separation as a function of radius for the galaxy model for different $N$.

over component value have to be chosen. Clearly, the single value of $\varepsilon$ will resolve well only some intermediate region of the galaxy model, but at the same time, there will be regions subject to numerical heating and poor resolution.

As is shown by Athanassoula et al. (2000) in a compound system the force error basically stems from the densest component and depends on its mass fraction. In a galaxy model, high density regions are located at the centre and in the plane of the disc, while low density regions are found at the periphery, mostly in the external halo. Since we are interested in processes occurring in the disc component, this should be well resolved. Thus, we compute the particle density over the cylindrical volume of the disc, which encompasses all three components of the galaxy:

$\Delta N(r, r+\Delta r)=\sum_{i=1}^{3} \Delta N_{i}(r, r+\Delta r)$,

with

$\Delta N_{i}(r, r+\Delta r)=\frac{\Delta M_{i}(r, r+\Delta r)}{M_{i}} N_{i}$.

The total $\lambda(r)$ for our galaxy model for different $N$ is shown in Fig. 3. The volume of the disc was divided in 50 equal volume concentric annuli and the mean separation was calculated in each annulus using Eqs. (10) and (12). It is interesting that with increasing $N$, the variation of $\lambda$ shallows. Of course, the shape of these curves will depend on the mass distribution of a particular galaxy model.

\subsubsection{The time-step}

The accuracy of an integrator scheme is determined by the time-step, $\Delta t$, which is in turn constrained by some criteria, e.g. the Courant condition. If two particles become close to each other, their acceleration increases. In order to handle their orbits adequately, the time-step should be reduced. Most $N$-body codes implement variable time-steps to achieve both a better efficiency and accuracy. These codes use for each particle an individual time-step, with criteria based on its local velocity and/or acceleration, see for example Springel et al. (2001). However, for codes that use a single time-step value for the evolution of the whole system, the magnitude of $\Delta t$ should be carefully estimated. Because the maximum acceleration is constrained by the softening parameter and this is related to the number of particles, in choosing the time-step one has to consider the role of both parameters.

In order to find the value of the time-step which is required for the correct integration of the orbits of particles in the shell, one may use the Courant condition which requires knowing their mean velocity of motion. In a spherical system in equilibrium steady state the velocities of the particles are usually derived from the Gaussian velocity distribution. The velocity dispersions, that are functions of the radius, can be obtained by taking the second momentum of the collisionless Boltzmann equation (Binney \& Tremaine 1987). For an isotropic velocity distribution of a spherically symmetric system, $\sigma_{r}=\sigma_{\varphi}=\sigma_{\theta}$, with

$\sigma_{r}^{2}(r)=\frac{1}{\rho(r)} \int_{r}^{\infty} \rho\left(r^{\prime}\right) \frac{G M\left(<r^{\prime}\right)}{r^{\prime 2}} \mathrm{~d} r^{\prime}$,

where $\rho(r)$ is the density and $M\left(<r^{\prime}\right)$ is the mass inside a sphere of radius $r^{\prime}$. The mean square velocity of particles in a shell of thickness $\Delta r$, is

$\left\langle v^{2}(r, r+\Delta r)\right\rangle=3\left\langle\sigma_{r}^{2}(r, r+\Delta r)\right\rangle$,

with

$\left\langle\sigma_{r}^{2}(r, r+\Delta r)\right\rangle=\frac{1}{\Delta M(r, r+\Delta r)} \int_{r}^{r+\Delta r} \sigma_{r}^{2}(r) \rho(r) \mathrm{d}^{3} r$.

The time required to travel the distance $\lambda$ in that shell is

$\langle\Delta t(r, r+\Delta r)\rangle=\frac{\lambda(r, r+\Delta r)}{\langle v(r, r+\Delta r)\rangle}$.

The space resolution is defined by the radius $R_{\text {res }}$, and the minimum time-step may be taken as the one estimated at $R_{\text {res }}$. Also, one can average the value of $\langle\Delta t(r, r+\Delta r)\rangle$ over the shells, obtaining the corresponding to the mean velocity of the system. This average time can be used as an estimate of the time-step for the orbit integrator. As a consequence, the central region having too short dynamical times will not be resolved.

For our galaxy model the maximum velocity is determined by the rotation curve $\left(\sim 250 \mathrm{~km} \mathrm{~s}^{-1}\right)$ and the velocity averaged over components varies with radius between 160 and $120 \mathrm{~km} \mathrm{~s}^{-1}$.

Though in galaxy simulations one uses Newtonian dynamics, the finite propagation speed of the gravitational interaction should be included for a realistic self-consist model. If gravity propagates with the speed of light $c$, then information about a particle on the opposite side of the system of radius $R=150 \mathrm{kpc}$, will be received after $\Delta t=2 R / c \approx 10^{6}$ years, or $\sim 1 / 250$ in our units. This implies a restriction on the minimum allowed time-step within which the information is received by all particles, and/or the maximum radius of the system that can be considered in order to maintain the consistency 
of the simulation. However, it can be shown that on galactic scales the error introduced by particles exterior to $c \Delta t$ is not greater than errors caused by the force approximation. The Newtonian approach can either be used when a steady state has been reached, because then the number of particles in a volume element maintains roughly the same, or when the system is not too extended and contribution from distant particles can be neglected. For a non-equilibrium rapidly evolving system, such as a cluster of galaxies, retarded gravitational potentials should be used.

In addition, the range of $\Delta t$ is restricted from a numerical point of view, by the requirement of stability of the integrator algorithm and the required accuracy.

Thus, the expressions given by Eqs. (10) and (17) permit us to define suitable values for the mean interparticle separation and the time-step as a function of the system's radius. The advantage is that they can be calculated during the construction of the model with the number of particles as an input parameter. It is clear that the selection of a single value for $\varepsilon$ and $\Delta t$ is not obvious. In what follows we will investigate the relation between the triad of parameters $(N, \varepsilon, \Delta t)$ and how their particular values affect the relaxation of the galaxy model.

\section{Numerical tests of galaxy relaxation}

In order to investigate the relaxation and stability against bar formation for various values of $\varepsilon, \Delta t$, and $N$, the isolated models were evolved up to $t=12.0$, which corresponds to $3 \mathrm{Gyr}$. The main results are summarized in Table 2, where the first column gives the model name, the second the total number of particles, the third the gravitational softening length, and the fourth contains the time-step. The rest of the columns represent our results, in the form of control parameters that are described below. There are eight series of runs. Models A01-A06 are performed with different $\varepsilon$ values that were chosen from Fig. 3 at uniform intervals. Models A07-A10 and A16-A23 are similar to the first series but for different $N$. The effect of the time-step is studied in models A11-A15. The time-steps are decreased by a factor of two, covering the range of velocities $0.64-10$ that satisfy the Courant condition for $\varepsilon=0.01$. Finally, models A24-A31 are performed using GADGET with different spline softenings in order to compare two forms of the force calculation.

The main parameter we use to measure the heating rate of the disc is the relative change of the velocity dispersion components. The averaged change in the velocity dispersion is defined over $n$ annulus as:

$\gamma_{k}^{2}=\frac{1}{n} \sum_{i=1}^{n}\left(\frac{\sigma_{k t_{2}}^{2}(i)-\sigma_{k t_{1}}^{2}(i)}{\sigma_{k t_{1}}^{2}(i)}\right)^{2}$,

where $\sigma_{k t_{2}}^{2}(i)$ and $\sigma_{k t_{1}}^{2}(i)$ are the $k$ th component of the velocity dispersions averaged over the $i$ th annulus at time $t_{2}$ and $t_{1}$, respectively, and $k=[r, \varphi, z]$. Their values are presented in Cols. 5-7 of Table 2. In absence of the heating process, these quantities should all be near zero, while non-zero values indicate an increment in particle velocity dispersions. The system should be relaxed towards a new equilibrium, because the initial models are only in approximate equilibrium and because of the introduction of the softening. For this we exclude the transient time interval $t<0.5 \mathrm{Gyr}$. We have evaluated $\sigma_{k}^{2}$ at times $t_{1}=0.5 \mathrm{Gyr}$ and $t_{2}=3 \mathrm{Gyr}$ which gives the accumulative estimation of the disc's anisotropic "temperature". Accordingly, the values of the relative change of the velocity dispersion were averaged over $n=16$ annuli. The value of the softening that minimizes these quantities will be taken as optimal.

For all test runs some disc angular momentum loss is observed. The transfer rate of angular momentum from the disc to the spherical components is related with the efficiency of dynamical friction. For a collisionless system in equilibrium, redistribution of angular momentum is undesired and should be minimized. The eighth column of the table shows, in percentage, the relative change of angular momentum with respect to the initial value, $L_{\mathrm{d} 0}$.

The stability of an infinitely thin rotating stellar disc to local axisymmetric perturbations is characterized by the Toomre parameter $Q=\sigma_{r} k / 3.36 G \Sigma$, where $\Sigma$ is the disc surface density (Toomre 1964). Discs with $Q<1$ are found to be subject to spontaneous bar formation, otherwise axisymmetric perturbations are suppressed. Moreover, non-axisymmetric perturbations in a differentially rotating disc of finite thickness can be suppressed if the critical radial velocity dispersion is roughly twice the Toomre critical velocity dispersion (Morozov 1981). In addition, the susceptibility of a thin stellar disc to swing amplification of the $m=2$ mode is characterized by the $X_{m}=$ $r \kappa^{2} / 2 m \pi G \Sigma$ parameter (Toomre 1981). The necessary condition to prevent the model from spontaneous bar growth is $Q \geq 2$ (Athanassoula \& Sellwood 1986), and to prevent swing amplification in galaxies with steep rotation curves, Mihos et al. (1997) found that $X_{2}>3$ is required. Their values in our initial models have a minimum of 1.65 and 3.25, respectively, and their final values for each run are shown in columns 9 th and 10th of Table 2 .

In order to detect the presence of a bar we use the so-called distortion parameter, defined as (Shibata et al. 2003):

$\eta=\sqrt{\eta_{+}^{2}+\eta_{\times}^{2}}$

where

$\eta_{+}=\frac{I_{x x}-I_{y y}}{I_{x x}+I_{y y}}, \quad \eta_{\times}=\frac{2 I_{x y}}{I_{x x}+I_{y y}}$,

and the moments of inertia are given by

$I_{i j}=\sum_{k=1}^{N_{\mathrm{d}}} m_{k} x_{k}^{i} x_{k}^{j}, \quad i, j=(x, y)$.

This parameter allows us to detect any non-axisymmetric deformations such as bars in the disc plane. The mean values of $\eta$ for each run are summarized in the 11th column of the same table. We have found traces of a bar for $\bar{\eta} \gtrsim 0.02$, which represents a rough threshold.

As criteria to estimate global errors in the force calculation, we use the conservation of the total energy, $E$, and the total angular momentum, $L$, whose percentage relative errors are also displayed in Table 2 (Cols. 12 and 13, respectively). 
Table 2. Numerical parameters of galaxy test runs. Tabulated are the model label (1), and the input parameters: the number of particles (2), the softening parameter $\varepsilon$ (3), and the time step $\Delta t$ (4). The units of length and time are $40 \mathrm{kpc}$ and $250 \mathrm{Myr}$, respectively. As a result of simulations the following control parameters are displayed: the relative change of components of the disc velocity dispersions (5-7), the disc angular momentum loss (8), the Toomre's $Q$ parameter (9), the Toomre's $X$ parameter (10), the average value of the distortion parameter (11), the conservation of the total energy (12) and the total angular momentum (13) of the system. Finally, we mention the code used (14).

\begin{tabular}{|c|c|c|c|c|c|c|c|c|c|c|c|c|c|}
\hline (1) & (2) & (3) & (4) & (5) & (6) & (7) & (8) & (9) & (10) & (11) & (12) & (13) & (14) \\
\hline Model & $N$ & $\varepsilon$ & $\Delta t$ & $\gamma_{r}$ & $\gamma_{\varphi}$ & $\gamma_{z}$ & $\frac{\Delta L_{\mathrm{d}}}{L_{\mathrm{d} 0}}, \%$ & $Q$ & $X_{2}$ & $\bar{\eta}$ & $\frac{\Delta E}{E_{0}}, \%$ & $\frac{\Delta L}{L_{0}}, \%$ & Code \\
\hline$\overline{\mathrm{A} 01}$ & 40960 & 0.025 & $1 / 512$ & 0.782 & 0.452 & 0.815 & 1.5 & 2.0 & 2.0 & 0.065 & 0.01 & 0.13 & $\overline{\text { GBSPH }}$ \\
\hline $\mathrm{A} 02$ & - & 0.020 & $1 / 512$ & 0.670 & 0.623 & 1.115 & 2.1 & 2.1 & 2.3 & 0.033 & 0.01 & 0.05 & - \\
\hline A03 & - & 0.015 & $1 / 512$ & 0.806 & 0.792 & 1.089 & 2.4 & 2.5 & 1.5 & 0.036 & 0.01 & 0.12 & - \\
\hline A04 & - & 0.010 & $1 / 512$ & 0.824 & 0.605 & 1.131 & 3.2 & 2.5 & 1.0 & 0.029 & 0.03 & 0.19 & - \\
\hline A05 & - & 0.005 & $1 / 512$ & 0.863 & 0.676 & 1.249 & 4.5 & 2.8 & 0.9 & 0.026 & 0.04 & 0.19 & - \\
\hline A06 & - & 0.001 & $1 / 512$ & 0.817 & 0.866 & 1.374 & 6.4 & 3.0 & 0.8 & 0.025 & 2.13 & 0.12 & - \\
\hline A07 & 163840 & 0.015 & $1 / 512$ & 0.457 & 0.335 & 0.446 & 0.8 & 1.9 & 2.5 & 0.021 & 0.01 & 0.02 & - \\
\hline A08 & - & 0.010 & $1 / 512$ & 0.541 & 0.443 & 0.532 & 1.0 & 2.2 & 2.5 & 0.019 & 0.01 & 0.02 & - \\
\hline A09 & - & 0.005 & $1 / 512$ & 0.573 & 0.417 & 0.662 & 1.3 & 2.3 & 2.0 & 0.015 & 0.02 & 0.05 & - \\
\hline A10 & - & 0.001 & $1 / 512$ & 0.679 & 0.556 & 0.909 & 2.8 & 2.6 & 1.5 & 0.016 & 0.57 & 0.03 & - \\
\hline$\overline{\mathrm{A} 11}$ & - & 0.010 & $1 / 64$ & 0.562 & 0.398 & 0.477 & 1.2 & 2.1 & 2.0 & 0.019 & 1.08 & 0.07 & - \\
\hline A 12 & - & 0.010 & $1 / 128$ & 0.527 & 0.397 & 0.466 & 1.0 & 2.1 & 2.0 & 0.017 & 0.04 & 0.05 & - \\
\hline $\mathrm{A} 13$ & - & 0.010 & $1 / 256$ & 0.536 & 0.462 & 0.470 & 1.1 & 2.2 & 2.5 & 0.019 & 0.02 & 0.02 & - \\
\hline A 14 & - & 0.010 & $1 / 512$ & 0.541 & 0.443 & 0.532 & 1.0 & 2.2 & 2.5 & 0.019 & 0.01 & 0.02 & - \\
\hline A 15 & - & 0.010 & $1 / 1024$ & 0.523 & 0.482 & 0.478 & 1.2 & 2.2 & 2.2 & 0.025 & 0.02 & 0.05 & - \\
\hline$\overline{\mathrm{A} 16}$ & 491520 & 0.010 & $1 / 512$ & 0.347 & 0.269 & 0.195 & 0.6 & 1.9 & 2.6 & 0.016 & 0.01 & 0.04 & - \\
\hline A17 & - & 0.008 & $1 / 512$ & 0.386 & 0.224 & 0.263 & 0.5 & 2.0 & 2.8 & 0.012 & 0.01 & 0.02 & - \\
\hline A18 & - & 0.006 & $1 / 512$ & 0.384 & 0.270 & 0.277 & 0.6 & 2.0 & 2.8 & 0.011 & 0.01 & 0.02 & - \\
\hline A19 & - & 0.004 & $1 / 512$ & 0.340 & 0.277 & 0.316 & 0.7 & 2.1 & 2.8 & 0.009 & 0.02 & 0.02 & - \\
\hline A 20 & - & 0.002 & $1 / 512$ & 0.333 & 0.253 & 0.382 & 0.7 & 2.2 & 2.7 & 0.008 & 0.04 & 0.03 & - \\
\hline A 21 & - & 0.001 & $1 / 512$ & 0.315 & 0.256 & 0.455 & 0.8 & 2.2 & 2.6 & 0.008 & 0.21 & 0.03 & - \\
\hline$\overline{\mathrm{A} 22}$ & 655360 & 0.008 & $1 / 512$ & 0.320 & 0.247 & 0.155 & 0.4 & 1.9 & 2.8 & 0.010 & 0.02 & 0.01 & - \\
\hline $\mathrm{A} 23$ & - & 0.001 & $1 / 512$ & 0.306 & 0.253 & 0.389 & 0.7 & 2.1 & 2.5 & 0.009 & 0.12 & 0.02 & - \\
\hline$\overline{\mathrm{A} 24}$ & 163840 & 0.015 & $1 / 512$ & 0.786 & 0.618 & 0.536 & 2.0 & 2.4 & 2.4 & 0.035 & 0.09 & 0.02 & $\overline{\text { GADGET }}$ \\
\hline A 25 & - & 0.010 & $1 / 512$ & 0.861 & 0.648 & 0.566 & 2.6 & 2.5 & 2.1 & 0.030 & 0.12 & 0.06 & - \\
\hline A26 & - & 0.005 & $1 / 512$ & 0.600 & 0.477 & 0.708 & 2.0 & 2.5 & 1.9 & 0.016 & 0.13 & 0.05 & - \\
\hline A 27 & - & 0.001 & $1 / 512$ & 0.658 & 0.529 & 0.908 & 2.5 & 2.6 & 1.4 & 0.014 & 0.79 & 0.05 & - \\
\hline $\bar{A} 28$ & 655360 & 0.008 & $1 / 512$ & 0.674 & 0.505 & 0.271 & 1.3 & 2.1 & 2.6 & 0.018 & 0.21 & 0.57 & - \\
\hline A29 & - & 0.001 & $1 / 512$ & 0.308 & 0.276 & 0.425 & 0.6 & 2.2 & 2.7 & 0.008 & 0.30 & 0.42 & - \\
\hline A30 & 1310720 & 0.006 & $1 / 512$ & 0.369 & 0.276 & 0.141 & 0.3 & 2.0 & 3.1 & 0.010 & 0.11 & 0.29 & - \\
\hline A31 & - & 0.001 & $1 / 512$ & 0.267 & 0.229 & 0.312 & 0.5 & 2.1 & 2.8 & 0.008 & 0.13 & 1.11 & - \\
\hline
\end{tabular}

In general, the models are quite stable and become dynamically "hot" $(Q \geq 2)$ which should prevent them from spontaneous bar formation. However, at the same time $X_{2}$ is reduced, making the disc susceptible to bar mode amplification. The major disc heating indeed occurs for $t<0.25 \mathrm{Gyr}$, while the system shifts to a new equilibrium; later on, the velocity dispersions grow relatively slowly. From Table 2 a general tendency of $Q$ to decrease linearly with increasing $\varepsilon$ can be observed for the Plummer softening.

As can be seen from Table 2, in models A01-A10, the variation of $\varepsilon$ affects most of the control parameters. There is a clear tendency of the parameters $\gamma_{r}, \gamma_{\phi}$ and $\gamma_{z}$ to increase with decreasing $\varepsilon$, caused by the heating process of the disc. The tendency is pronounced for small $N$, and weakens for large $N$ (models A16-A23). Indeed, no simple interpretation of the behaviour of $\gamma_{r}$ and $\gamma_{\phi}$ on $\varepsilon$ can be drawn for the simulations with higher number of particles (models A16-A23) and those performed with GADGET (models A24-A31). However, it is clear that with the increasing $N$ the heating is reduced.

During the evolution, there are some halo particles that escape from the system, but their number is relatively small ( $<2$ per cent), and for all models the density profile of the spherical components is well preserved. For small values of $\varepsilon$, the disc rapidly heats up, and reaches very large values 
of $Q(>2)$. In these cases, we observe suppression of a transient spiral pattern after a few rotation periods, indicating the presence of dynamical heating of the disc. For large $\varepsilon$ the central region of the galaxy model with $N=40960$ rapidly collapses, and a ring-like structure moving outwards is observed during the first few time steps. The latter is due to the non-equilibrium evolution of the model caused by the lowered potential energy and the strong deviation from the Newtonian law. Regarding the disc vertical structure, it is observed that for smaller $\varepsilon$, discs become thicker during the evolution. The two-body relaxation effects in the vertical direction become important as the vertical structure is being resolved. This makes the disc hotter and thicker (compare $\gamma_{z}$ values in Table 2).

We allowed some models to run up to $t=8 \mathrm{Gyr}$, at which $\eta$ reaches the value $\sim 0.1$. Since $t \approx 4 \mathrm{Gyr}$ we found a weak diffuse bar, that appears even for relatively high values of $Q(>2.0)$, that indicates that such high $Q$-values alone cannot suppress the bar instability. A more massive halo also prevents the bar formation for a longer time. However, Athanassoula (2003) has recently shown that a live halo can play a rather destabilizing role. The time at which the bar forms varies slightly with $N$ and $\varepsilon$, appearing earlier for small $N$ and large $\varepsilon$ (see values of $\bar{\eta}$ of the Table 2). This confirms findings of Walker et al. (1996), who showed that the bar emerges later when $N$ is increased, and for a rigid halo the bar does not form. Large softenings lead to formation of a bar that is more pronounced, and appears at earlier times $(t \approx 2$ Gyr $)$ in spline softening models than in Plummer's.

To demonstrate that the total energy conservation mainly depends on the time-step, we vary $\Delta t$ and fix the rest of the numerical parameters constant. This is done in models A11-A15, which show that the main change among the control parameters is in the energy. The deterioration of energy conservation for smaller gravitational softenings is explained by the errors introduced by the numerical leap-frog integrator for particles with high accelerations which are integrated with large timesteps. When the softening is small but the time-step is sufficiently large, such as for example in model A06, a systematic non-conservation of the total energy is observed at each timestep. As shown in models A11-A15, for $\varepsilon=0.01$ and for typical velocities $\bar{v} \sim 1$ or $\sim 156 \mathrm{~km} \mathrm{~s}^{-1}$, a time-step of $1 / 128$ is quite sufficient to obtain energy conservation of $\sim 0.04$ per cent after $3 \mathrm{Gyr}$ of evolution. On the other hand, comparing the energy conservation between models with $\varepsilon=0.001$, it can be noted that the increase of $N$ also improves energy conservation. This is probably due to the accomplishment of a smoother gravitational field.

For comparison, we have performed several runs using the GADGET code (models A24-A31). We have decided to skip the runs with $N=491520$ but preformed instead the simulations with $N=1310720$. These runs, in general, show higher heating rates and poorer energy conservation. The higher heating rate of these models suggests that the Plummer softening makes the system less collisional than the corresponding spline softening. This is due to a slower convergence of the Plummer model to the Newtonian force (Theis 1998).

The high particle numbers of models A16-A21 and A22-A23 decrease the overall process of dynamical

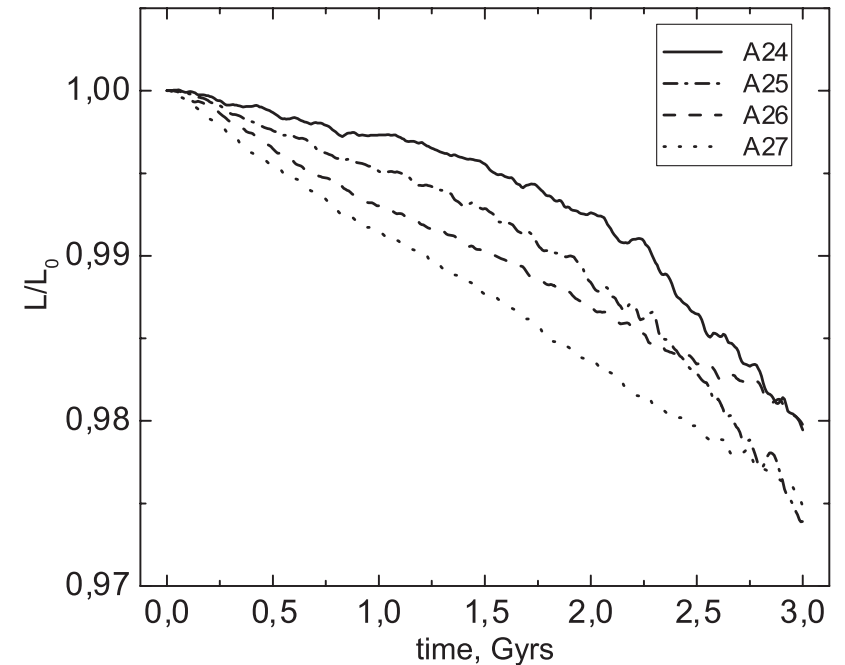

Fig. 4. Evolution of the disc angular momentum for models A24-A27, normalized to the initial value.

heating and diminish the distortions of the disc surface density. By comparing the $\gamma$ values with the same $\varepsilon$ and different number of particles, we found that augmenting $N$ by four times, the $\gamma$ values diminish few times. For the given range of $\varepsilon$ in models A16-A21, the weak variation of most of their control parameters indicates that they are close to convergence. Models A22-A23 confirm this convergency. Models A30-A31 that were performed using GADGET code also show further reduction of the disc heating and give results comparable to those of models A22-A23, which were performed with GBSPH code. Note also, that these models show no signs of a bar.

The effect of the softening clearly manifests itself in the amount of angular momentum transferred from the disc to the bulge and halo. The eighth column of Table 2 shows the dependence of the disc's angular momentum loss on the numerical parameters. In all runs decreasing the Plummer softening, increases the disc angular momentum loss. The observed loss rate is almost constant through a single simulation and is different for each run. For spline softening (models A24-A31) this process is slightly different, as can be seen in Fig. 4. The loss rate is linear in models A26 and A27 as for models with the Plummer softening, but in models A24 and A25, as well as in A28, the bars emerge quite rapidly at $t \approx 2 \mathrm{Gyr}$, which increases the growth rate. Concerning the total angular momentum, it can be noted that it is conserved quite well and almost independent on our chosen numerical parameters.

The numerical tests have shown that for the models in Table 2, the softening $\varepsilon=0.001$, for the considered range of $N$ and $\Delta t$, has the higher heating rate and the worst total energy conservation, but, at the same time, smaller non-axisymmetric distortions. This value probably fulfills a criteria similar to the ones given through Eqs. (5) and (6) for a compound galaxy model and minimize the initial force errors. However, these models cannot be accounted as realistic ones (except for the model A31), but, for comparison, we will use them in some of the collisions described in the next section. Other extreme cases are those that use $\varepsilon$ evaluated at the edge of the disc. 
Despite the fact that in some models they do not resolve the inner and vertical structure of the disc, but only its outer part, they show the smallest heating and a good conservation of $E$ and $L$. These models are also used in collision runs. From Table 2 we choose as compromise values for $\varepsilon_{\text {opt }}$ the ones that correspond to models A03, A08 and A19. In addition, the models A22-A23 and A28-A29 have also been taken since they show convergence in the control parameters.

\section{Galaxy collisions}

In this section we describe the effect of the number of particles and the gravitational softening on the properties of an interacting system. Galaxy models are constructed with different $\varepsilon$ and $N$ values and used in galaxy collision simulations to study the effect of $\varepsilon$ on the formation of bars and their properties, such as the amplitude and rotational velocity. In order to reduce the parameters' space, all collision simulations were performed with the single time-step $\Delta t=1 / 256$.

For close galaxy encounters, interaction between particles are much more important than for an isolated galaxy. The value of $\varepsilon_{\text {opt }}$ found for an isolated steady state system is not necessarily optimal for an interacting system where strong non-linear effects and large density gradients are developed. Force errors and relaxation processes are drastically increased by the encounter due to the almost head-on collision of particles along the shock front. Moreover, the time-step found for the isolated galaxy models may be inadequate for collision simulations.

For the simulations, equal galaxies are placed on parabolic orbits, calculated from the two-body problem with time to pericentre $t_{\mathrm{p}}=0.75 \mathrm{Gyr}$ and pericentric separation $p=2 R_{\mathrm{d}}=$ $32 \mathrm{kpc}$. The latter is chosen such that at the pericentre the discs are just touching each other, and the main perturbation is provided by the interaction of the extended haloes. This pericentric separation provides enough time for the bars to evolve and, at the same time, to investigate the merger process. With such orbital parameters, the galaxies are initially separated by $R_{\mathrm{p}} \approx 160 \mathrm{kpc}$, which sets the system with overlapped haloes and thus, it introduces some initial perturbations. The relatively small $R_{\mathrm{p}}$ is chosen to reduce cumulative numerical errors, where the preferred duration of interaction should be as small as possible. The galaxies were relaxed up to $t=0.25 \mathrm{Gyr}$ before placed on their orbits.

We only consider prograde-retrograde collisions that allows us to investigate, with a single configuration, bar formation processes in the two possible directions of rotation. Planar collisions (i.e., disc planes of the galaxies coincide) are chosen, because this configuration is the most violent and provides the maximum perturbation and maximum transfer of orbital to internal angular momentum of the discs. In the present study we do not pretend to cover the entire orbital parameters space. The emphasis is rather to investigate the influence of the numerical parameters on the bar formation for a given configuration.

Details of collision models and conservation of total energy and angular momentum are summarized in Table 3. Animations of some collision simulations are available at www. astro.inin.mx/ruslan/tidal_bars
Table 3. Parameters of collisions. The units of time and length are $250 \mathrm{Myr}$ and $40 \mathrm{kpc}$, respectively.

\begin{tabular}{lcrcrcc}
\hline \hline Model & $N$ & $\Delta t$ & $\varepsilon$ & $\frac{\Delta E}{E_{0}}, \%$ & $\frac{\Delta L}{L_{0}}, \%$ & Code \\
\hline B01 & 40960 & $1 / 256$ & 0.001 & 14.53 & 0.06 & GBSPH \\
B02 & - & $1 / 256$ & 0.015 & 0.09 & 0.10 & - \\
B03 & - & $1 / 256$ & 0.025 & 0.14 & 0.40 & - \\
\hline B04 & 163840 & $1 / 256$ & 0.001 & 5.40 & 0.05 & - \\
B05 & - & $1 / 256$ & 0.010 & 0.11 & 0.10 & - \\
B06 & - & $1 / 256$ & 0.015 & 0.10 & 0.16 & - \\
\hline B07 & 491520 & $1 / 256$ & 0.001 & 2.17 & 0.07 & - \\
B08 & - & $1 / 256$ & 0.004 & 0.10 & 0.16 & - \\
B09 & - & $1 / 256$ & 0.010 & 0.09 & 0.11 & - \\
\hline B10 & 655360 & $1 / 256$ & 0.001 & 1.61 & 0.06 & - \\
B11 & - & $1 / 256$ & 0.008 & 0.07 & 0.08 & - \\
\hline B12 & 163840 & $1 / 256$ & 0.001 & 7.0 & 4.98 & GADGET \\
B13 & - & $1 / 256$ & 0.010 & 0.33 & 5.87 & - \\
B14 & - & $1 / 256$ & 0.015 & 0.27 & 4.98 & - \\
\hline B15 & 655360 & $1 / 256$ & 0.001 & 2.17 & 2.94 & - \\
B16 & - & $1 / 256$ & 0.008 & 0.11 & 2.33 & \\
\hline
\end{tabular}

In order to perform a reliable analysis of the bar characteristics, its centre of mass should be well defined. The disc particles that are ejected to large distances can spuriously affect the parameters under study, and therefore these particles are excluded from the analysis. We then calculate the centre of the disc as follows: first we compute the centre of mass using all the particles of the disc. We then recalculate a new centre of mass with only the disc particles that are located inside $2 R_{\mathrm{d}}$ with respect to the firstly calculated centre of mass. The centre of mass found in such a way gives roughly the position of the centre of mass of the disc with respect to which the bar parameters are further computed. However, this procedure fails during the last stage of the collision process when both systems are found inside the same radius.

To characterize quantitatively the bar, we use two criteria. The first is given through the distortion parameter $\eta$ defined above. The second is to compute the harmonic amplitudes of the disc mass distribution in the equatorial plane, which indicate the presence of any non-axisymmetric deviation. The normalized coefficients are (Sellwood \& Athanassoula 1986)

$A_{m}=\frac{1}{n} \sum_{j=1}^{n} \exp \left(\mathrm{i} m \theta_{j}\right)$,

where $n$ is the number of particles within the radius of the disc $R_{\mathrm{d}}$, and $\theta_{j}$ is the polar angle of the $j$ th particle with respect to the centre of mass. Since we are mostly interested in bisymmetric perturbations, we set $m=2$, but we also investigate amplitudes up to $m=8$ to compare among the harmonics. We have found that the $\eta$ and $\left|A_{2}\right|$ bar strengths criteria give similar results. However, stronger fluctuations are observed using $\eta$, and in what follows we will only refer to $\left|A_{2}\right|$. Some morphological characteristics of the bar can be obtained from 


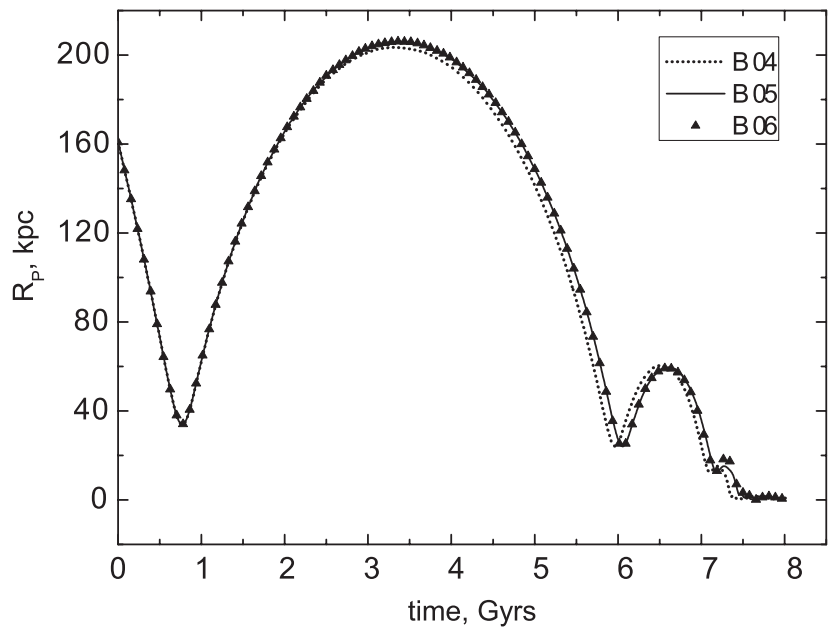

Fig. 5. The separation between the centres of the two discs as a function of time for models B04-B06.

projected density contours and projections of particles on the orthogonal planes.

An important quantity that characterizes the bar is its rotational velocity $\Omega$, that is given by the rate of change of the angle between the coordinate axis and the principal axis of the moment of inertia of the bar. We divide the plane of the disc into 16 concentric cylinders for which the eigenvalue of the moment of inertia tensor $I_{x x}$ is calculated. Then we iteratively rotate the coordinate system with an angular step of $2^{\circ}$ until the maximum value of $I_{x x}$ is reached for each annuli. The corresponding averaged polar angle of rotation $\phi$ with respect to the original coordinate system is taken as the phase of the bar. The procedure is repeated for each snapshot file, and $\Omega$ is found as $\mathrm{d} \phi / \mathrm{d} t$, where $\mathrm{d} t=1 / 32$ is the time interval between snapshots. To correctly identify the bar major axis, we impose the restriction on the minimum value $\eta_{+}=0.15$, which excludes the time intervals when the bar is not present and excludes also the central annulus, which is almost axisymmetric. The second restriction consists to discard annuli with a phase difference of more that $10^{\circ}$ from the averaged phase of the inner annuli. This procedure excludes the annuli where spiral arms and the ring are located.

For all galaxy collision models, the first encounter occurs at time $t \approx 0.8 \mathrm{Gyr}$ and the second at $t \approx 6 \mathrm{Gyr}$. The tidal interaction induces a bipolar perturbation that develops spiral arms in the first galaxy which is in prograde orbit, while the second galaxy does not show any significant deformation. The subsequent evolution is different for each model as will be described below.

Figure 5 shows the separation between the centres of the two discs as a function of time for models B04-B06. It can be noted that in model B04, after the first encounter, the discs have a maximum separation distance at $t \approx 3.5 \mathrm{Gyr}$, which is less than those of models B05 and B06 due to a smaller softening of the force. In addition, the whole collision process is slightly faster for model B04, and slower for B05 and B06. By analysing the curves for runs B01-B03 (not shown here) we have noted that they have a similar shape, and that by increasing $N$ (collisions B07-B09) the difference tends to vanish.

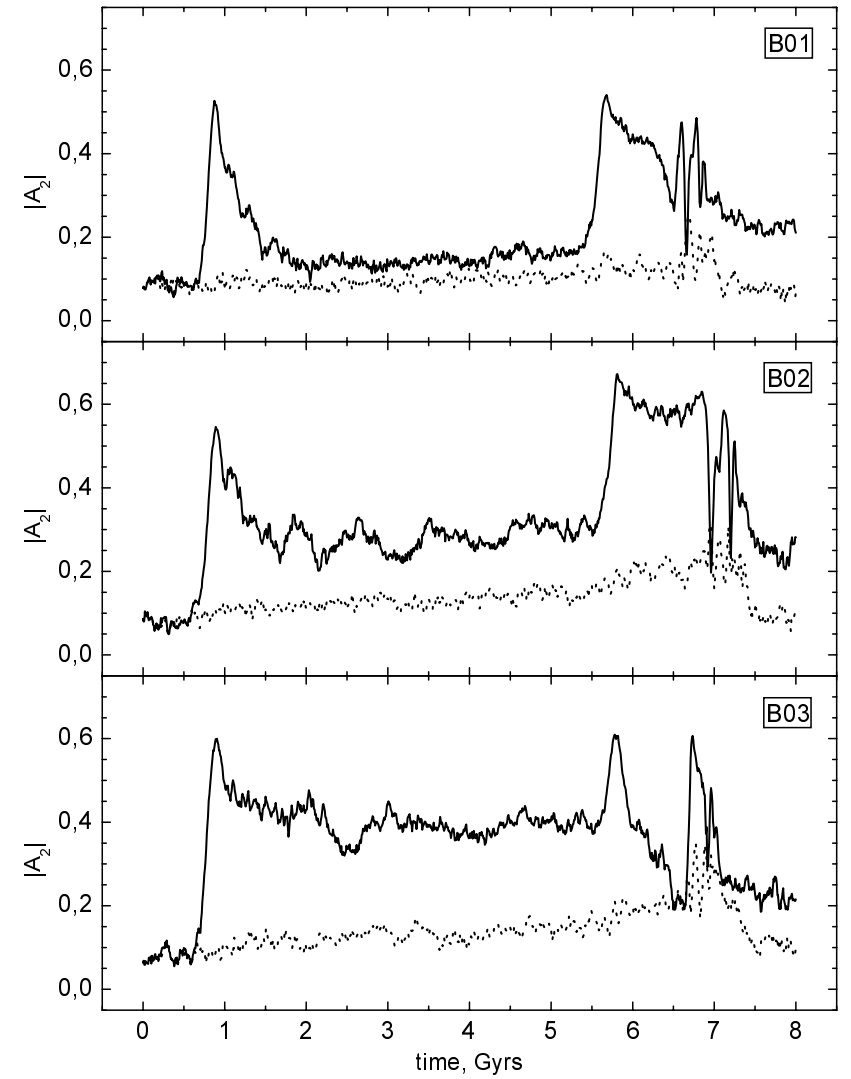

Fig. 6. The evolution of the amplitude of the second harmonic for models B01-B03. The solid line corresponds to the first galaxy (prograde orbit) whereas the dotted line corresponds to the second galaxy (retrograde orbit).

Models B01-B09 were performed with the GBSPH code with three different values of $N$ and $\varepsilon$. We first describe collision runs B01-B03 which were performed with $N=$ 40960 particles per galaxy. As can be seen from Table 3, the run B01 shows bad conservation of the total energy due to integration errors caused by inadequate time-step for such small $\varepsilon$. But at the same time, there is a good angular momentum conservation. The evolution of the bar amplitude for these runs is shown in Fig. 6 for both prograde (solid line) and retrograde (dotted line) discs. There are also small contributions from other harmonics, mostly from even ones.

After the first encounter, transient spiral arms are formed in the prograde galaxy which then become more and more tightly wound until they form a disc again. At the same time, a small diffuse bar emerges which maintains an oval shape of apparent length $l \sim 10 \mathrm{kpc}$ until the second collision, after which the bar is amplified to length $l \sim 16 \mathrm{kpc}$ and develops a butterfly shape when viewed from the disc's plane.

In model B03 after the first passage, transient open spiral pattern is generated in the first galaxy, and at the same time, a strong bar of length $l \sim 20 \mathrm{kpc}$ appears. Note that this is the simulation with the longest bar. The spiral arms begin to wind around the bar, forming a ring around it which is then slowly absorbed by the bar. The bar maintains its length until the second close approach, after which it is partially destroyed and reduced to $l \sim 16 \mathrm{kpc}$. 


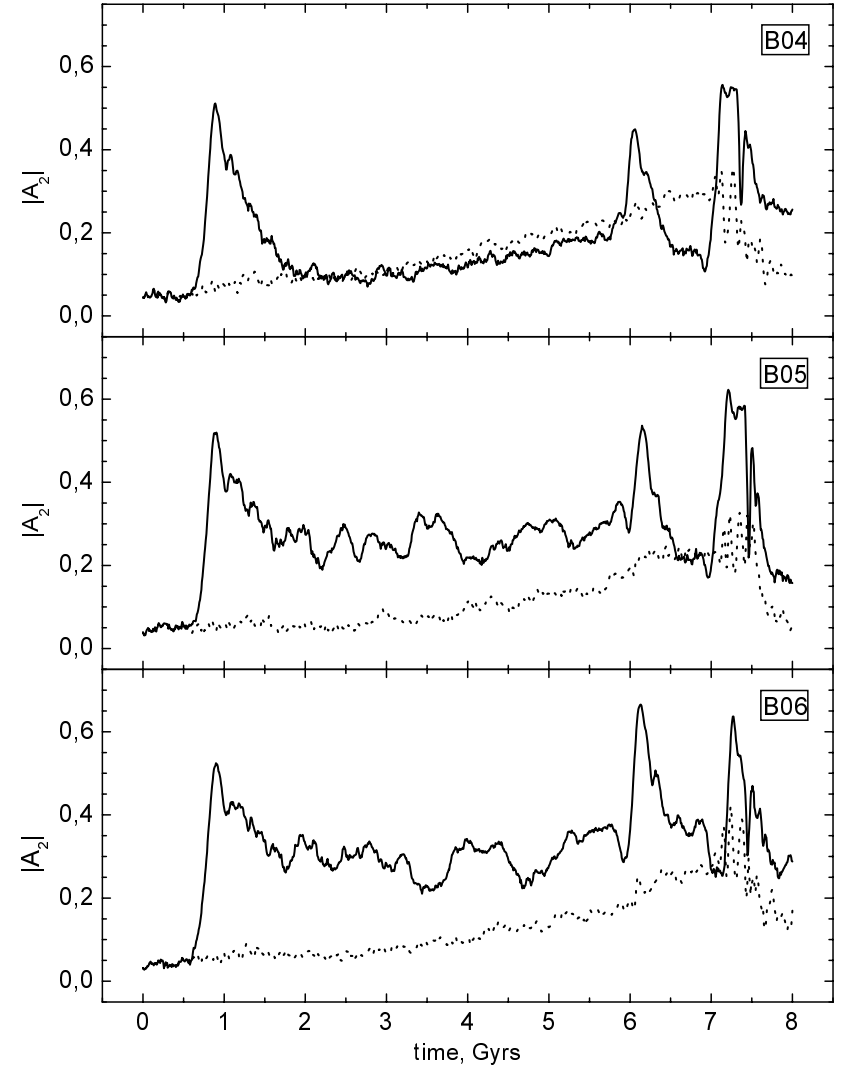

Fig. 7. The evolution of the amplitude of the second harmonic for models B04-B06. The correspondence of curves is the same as for the previous figure.

The second galaxy that is in retrograde motion shows a slowly growing bar mode, which is amplified by the second encounter (see Figs. 6-8). The barred galaxies collide again and finish in a disc-like remnant. The initial growing mode of the second galaxy that becomes notable after $t \approx 4 \mathrm{Gyr}$ is similar to the one in the isolated evolution, described in Sect. 3. Therefore, it seems not to be caused by the tidal interaction, but instead by the instability of the galaxy model. This is observed in all collision models presented in Table 3 . The instability growth rate is slow and by the time of the first encounter the bar has not yet been formed. However, the evolution of the bar amplitude after $t \approx 6 \mathrm{Gyr}$ may be influenced by both effects.

The behaviour of the B02 collision run, is similar to the B03, except that the bar is shorter $(l \sim 16 \mathrm{kpc})$. After the second collision, the bar is strongly amplified, because at the pericenter it becomes aligned with a diffuse bar of the second galaxy. The amplitude of the second harmonics of the bar reaches the value $\sim 0.6$, but apparent length of the bar does not change, obtaining rather a butterfly shape.

The models B04-B06 are performed with $N=$ 163840 particles per galaxy and show a behaviour similar to models B01-B03 but with richer details in the inner structure. For example, a $\S$-shaped structure surrounding the bar was present in these runs, but it was not observed in simulations with $N=40960$ particles. The notable tidal perturbation decay is also observed in model B04, but the difference between the models B05 and B06 is not so strong (Fig. 7). The

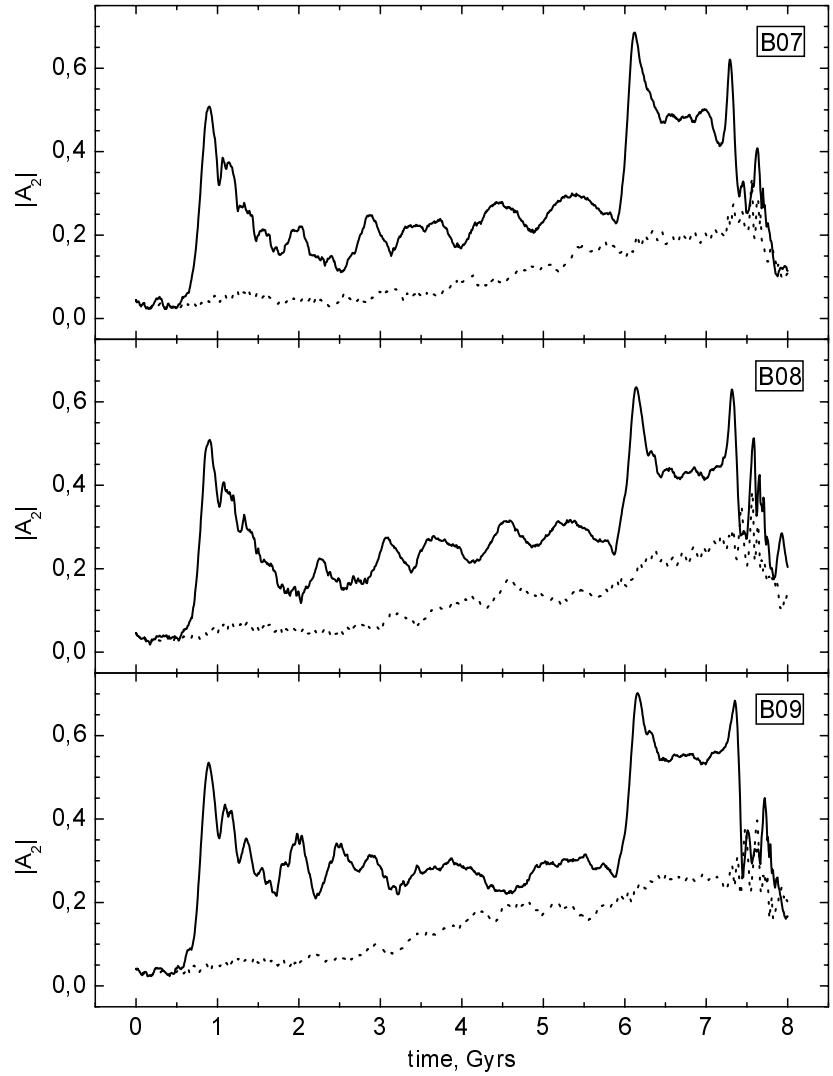

Fig. 8. The evolution of the amplitude of the second harmonic for models B07-B09. The correspondence of curves is the same as for Fig. 6.

models B07-B09 are performed with higher $N$ and show a more detailed inner structure, but only a weak dependence of bar strength on $\varepsilon$ (see Fig. 8).

Similar differences are observed in the rotational velocities of the bars. The evolution of bar angular velocities for models B01-B09 are plotted in Fig. 9. As it can be noted form the upper panel, the magnitude of $\Omega$ differs significantly among the runs. However, with the increase of $N$ these differences are reduced, and the shape of the curves becomes similar (models B07-B09). A clear evidence for correlation between the bar strength and its angular velocity can be seen when Figs. 6-9 are compared. For example, after the second encounter the decay of $\Omega$, observed in models B07-B09 occurs when the bar, due to alignment with the second bar, is amplified. This reduction can be associated with the angular momentum exchange which amplifies the bar and reduces its pattern speed, in accordance with results of Berentzen et al. (2004).

The softening affects not only the shape of the bars, but also its internal structure. Figures 10 and 11 present the contours of the projected density for models B04-B06 at different $t$. From the upper panels it can be seen that due to different $\varepsilon$, the initial discs have different central densities, which may be responsible for their subsequent evolution. The rest of the panels show that bars with smaller $\varepsilon$ are rounder, denser and smaller than those with larger $\varepsilon$.

Summarizing, the results of models B01-B09 have shown that the decrease of $\varepsilon$ damps perturbations in the prograde disc. 


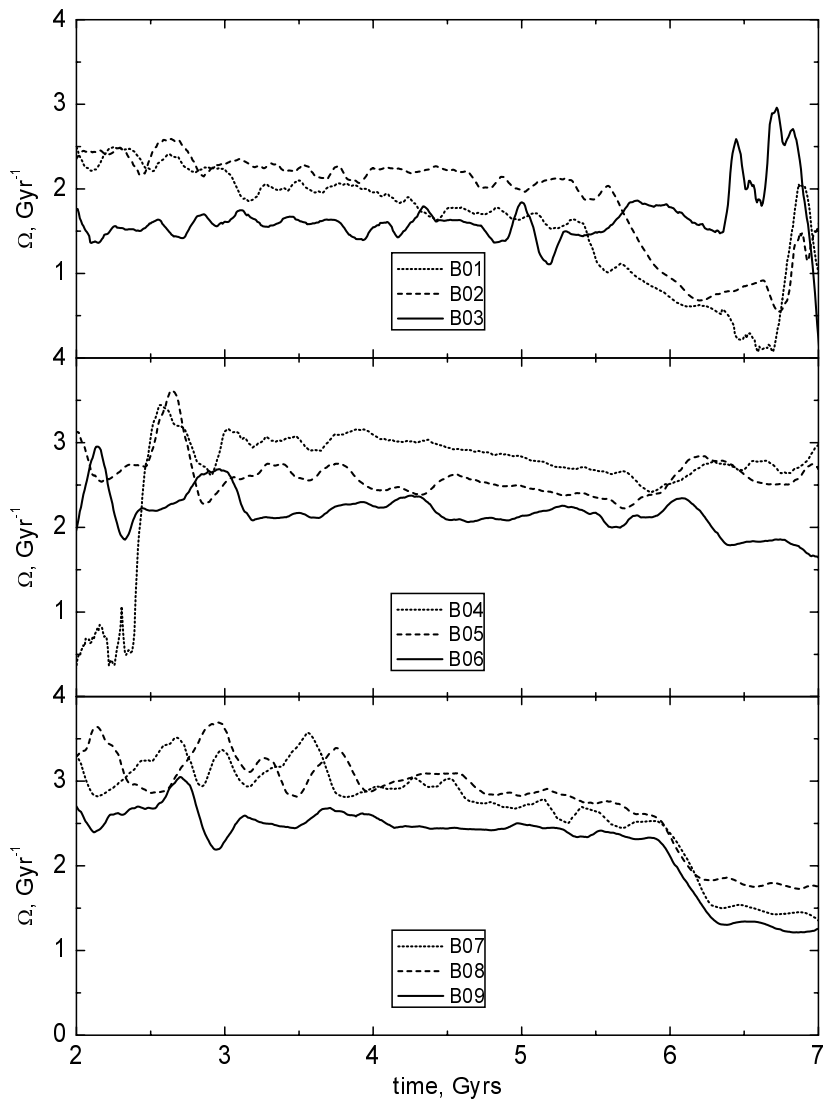

Fig. 9. The evolution of bar angular velocity for collision runs B01-B09. Shown only $\Omega$ of the first (direct) disc.

For the second galaxy which is retrograde, the amplitude $\left|A_{2}\right|$ slowly grows with time, and the growth rate slightly depends on the chosen $\varepsilon$. Figures 6-8 indicate that the initial perturbation has almost the same peak value of $\left|A_{2}\right| \gtrsim 0.5$, but further decay of tidal response depends on $\varepsilon$ and $N$.

For comparison, we have performed collision simulations using a parallel version of the GADGET code (runs B12-B16). As can be seen from Table 3, the total angular momentum is poorly conserved. After the first encounter, this nonconservation shifts the galaxies from the orbital plane, which is more notable for small $N$ (runs B12-B14). As a consequence, further encounters are not planar and the merger process takes longer. Despite this, the first stage of the bar formation can be compared with previous runs. Figure 12 shows the evolution of the amplitude of the second harmonic for collisions B12-B14, which is similar to models B04-B06 except that the amplitude of the bar is a bit smaller and a stronger bar develops in the second disc. The collision simulations performed using GBSPH and GADGET codes for $N=655360$ show no significant differences in $\left|A_{2}\right|$ for the same $\varepsilon$; these curves are not shown.

Finally, we will describe some peculiar characteristics of collision and merger processes for different softenings. A general interesting feature is observed between the first and the second collisions. The two large spiral arms that are formed just after the first collision in the prograde galaxy become more and more tightly wound, and form a ring-like structure that

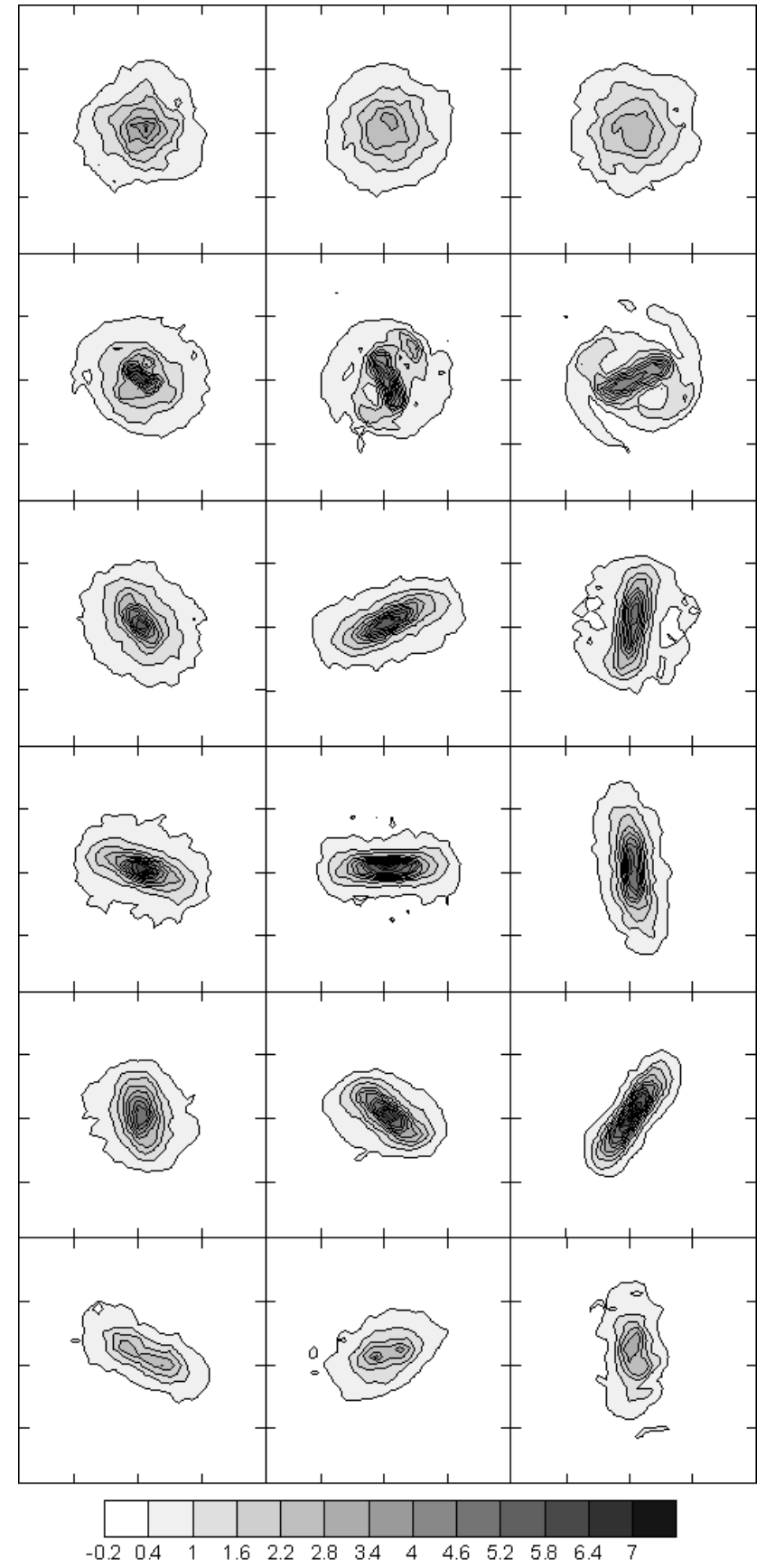

Fig. 10. Projected density contours of the first (prograde) disc in units of $137.5 M_{\odot} / \mathrm{pc}^{2}$. Columns from left to right correspond to models B04, B05 and B06. The boxes are $32 \times 32 \mathrm{kpc}$ long. Contours are shown for times (panels from top to bottom) 0, 1.5, 3.5, 5.5, 6.5 and $8 \mathrm{Gyr}$.

surrounds the diffuse bar. This ring is then slowly destroyed accreting into the bar, and short transient arms are formed at the extremes of the bar (Fig. 13). These arms change from trailing to leading and vice-versa with a period $T_{\text {arm }} \approx 0.675 \mathrm{Gyr}$ (model B06) though the direction of rotation of the bar does not change. We refer to this dynamical feature as the wiggle effect. As most particles are absorbed by the remaining pronounced bar, the wiggle slowly vanishes but does not completely disappear and is sustained until the second collision. The wiggle is 


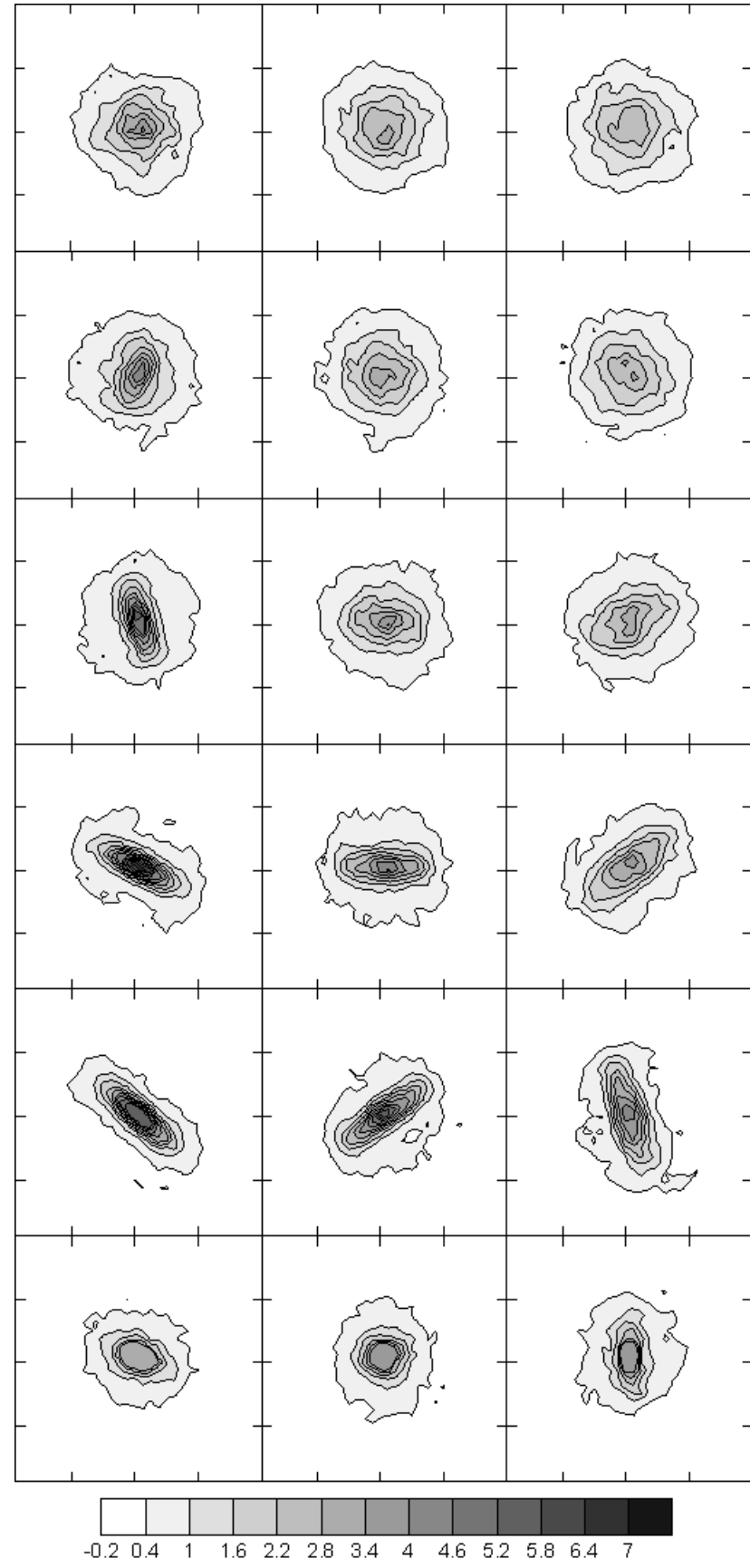

Fig. 11. Projected density contours of the second (retrograde) disc. Columns from left to right correspond to models B04, B05 and B06 respectively. All scales are the same as in the previous figure.

observed in runs where a clear bar appears and in all runs with $N=491$ 520. In the same figure, shown aside, a classical bar buckling leading to the box/peanut shape may be seen.

In our orbital configuration, after the first encounter up to 5 per cent of the particles of the first disc leave the initial radius, and up to 10 per cent after the second. The second disc looses only a few particles during the collision. The particle loss rate is almost independent on the numerical parameters and in each case the merger remnant is composed of roughly 70 and 85 per cent of particles of the first and second discs, respectively. In runs B01-B06 the remnants have elongated orbits due

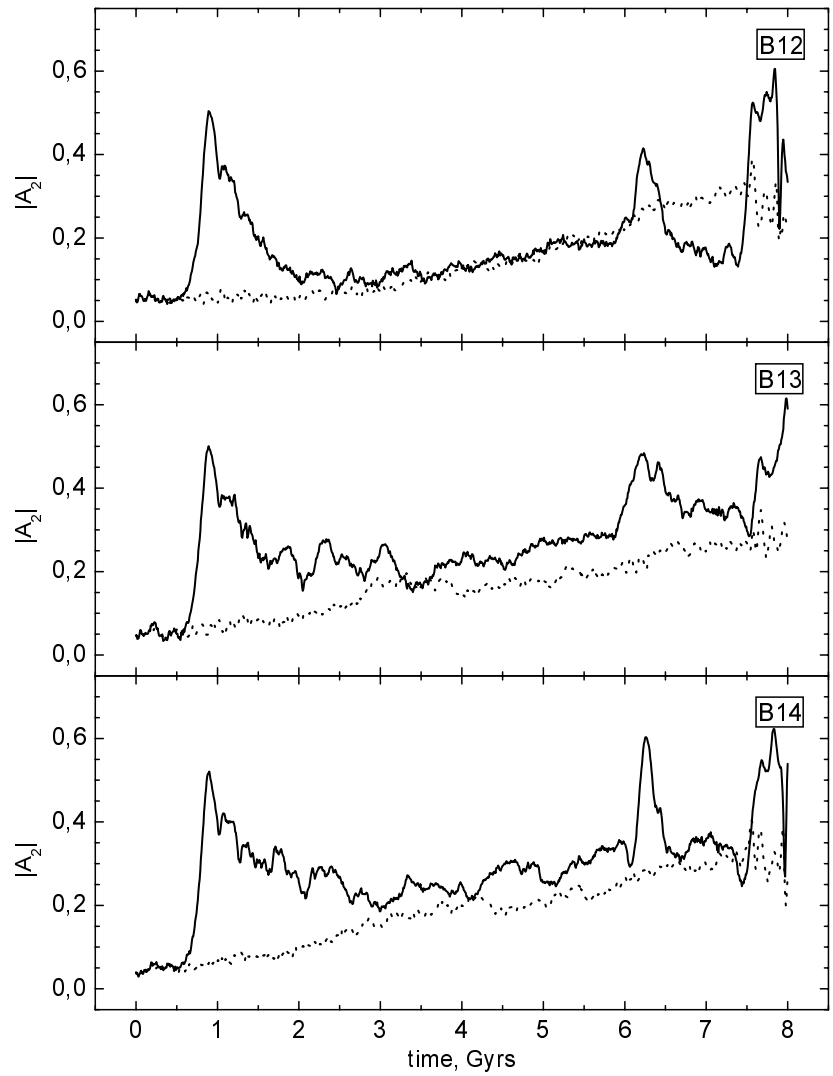

Fig. 12. The evolution of the amplitude of the second harmonic for models B12-B14. The correspondence of curves is the same as in the Fig. 6.

to particles of the prograde disc, whereas particles of the retrograde disc form a symmetric constant density core (Figs. 10, 11 bottom panel). The mergers with large softenings finish in a disc-like remnant, whereas for small softenings, the remnant has rather an elliptical structure.

\section{Discussion}

The aim of the convergence study was to investigate how the numerical parameters influence the relaxation and stability of a galaxy model against bar formation. We first discussed how the parameters $N, \varepsilon$ and $\Delta t$ can be restricted, and for a particular galaxy model obtained valid ranges for $\epsilon$ and $\Delta t$. For a galaxy model represented by a given number of particles, the local mean interparticle separation in the plane of the disc can be easily estimated. Although the gravitational softening parameter can be chosen to be equal to any particular value of $\lambda$ within the established range, one still has to make a compromise between force accuracy and relaxation. But it seems that there are no universal choices, and the selection of a particular $\varepsilon_{\mathrm{opt}}$ value for for a whole system depends on dynamical aspects one wants to study (Romeo 1998). In this context we wish to minimize the relaxation effects that are responsible for drifting of a system from its initial condition. The convergence study performed in Sect. 3 showed that relaxation processes are minimized for $\varepsilon$ equal to the maximum $\lambda\left(R_{\mathrm{d}}\right)$, which is evaluated at the edge of the disc. This $\varepsilon$ gives low heating and good energy 

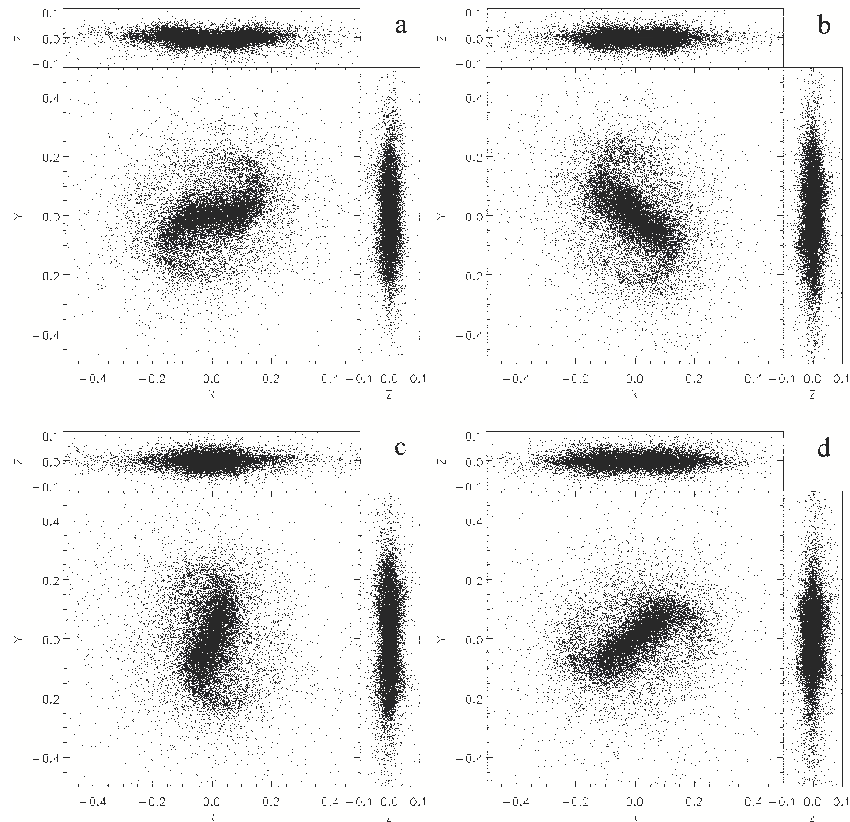

Fig. 13. Projection of particles of the first disc for run B06 for times: a) 3.125 , b) 3.875 , c) 4.375 and d) 5.125 Gyr. For face-on and edge-on projections the boxes are $40 \times 40 \mathrm{kpc}$ and $8 \times 40 \mathrm{kpc}$ long, respectively. The bar always rotates clockwise, but its arms wiggle from trailing to leading and vice-versa.

conservation, but it neither resolves the vertical nor the radial disc mass distributions. On the other hand, the minimum $\varepsilon=\lambda(0)$ is unacceptable in simulations because of the high heating rate, and because it demands a too small time-step to achieve acceptable energy conservation. In Table 2 we have presented a series of models with gravitational softenings chosen within the established range which is defined by $N$. Figure 3 indicates that the $\lambda$ range decreases with increasing $N$.

We have found that for our galaxy models the characteristics of the resulting systems tend to converge for $N \gtrsim 491520$. Namely, the reduction of the range of $\lambda$ by increasing $N$ also reduces the variation of control parameters. The parameters $\gamma_{z}$, $Q$ and the disc angular momentum loss rate, used to measure the disc heating, proved to be quite informative. The disc angular momentum loss rate gives the efficiency of the dynamical friction between the disc and the halo, whereas the increase of the vertical velocity dispersion, observed in all runs and for both softening methods, measures the thickening of the disc. However, it seems that those quantities are related. The dynamical friction is enhanced by increasing the thickness of the disc, and this, in turn, increases the angular momentum loss rate. Indeed, the change in the slope of angular momentum loss of the disc serves as a good indicator to identify the bar's emergence. As can be seen from Table 2, simulations with 491520 particles greatly reduce the disc angular momentum loss, which is less than 1 per cent within $3 \mathrm{Gyr}$, and only weakly depends on $\varepsilon$. The disc thickening is also reduced to about three times, when compared to runs with 40960 particles. Simulations with $N=1310720$ show that the heating process is weakly affected by $\varepsilon$ in comparison with the simulations with smaller particle numbers for the corresponding ranges of $\lambda$. From the same table it can be noted that final values of Toomre's parameter $Q$ for the Plummer softening show approximately a linear dependence on $\varepsilon$.

The heating of the disc can be reduced by increasing either the softening parameter or the number of particles. However, increasing $\varepsilon$ within the allowed range reduces the heating in a slower way than increasing $N$ (compare the values of $\gamma$ in the Table 2). This reaffirms the common belief that the increasing of the number of particles lets to effectively decrease the numerical effects and at the same time to increase the resolution. To obtain the minimum $N$ that resolves the disc vertical structure, it is necessary that $\varepsilon \lesssim z_{0}$. The criterion of the interaction radius, given through Eq. (5), showed to be inadequate because it gives too small $\varepsilon$ values. On the other hand, the criterion given through Eq. (11) would give a minimum $\varepsilon$, which is approximately 0.3 times the mean interparticle separation, estimated within the half-mass radius. The interaction force in this case will be well represented and the bar will appear later, but the system will be heated.

On the other hand, the time-step, which is proportional to the gravitational softening parameter, defines the accuracy of the integration of the orbits and is the main factor responsible for energy conservation. The criterion for the time step $\Delta t \lesssim \varepsilon / \bar{v}$ showed to be satisfactory, at least in simulations performed with the Plummer softening (GBSPH). For a fixed $N$ the energy conservation deteriorates when $\varepsilon$ is reduced. If $N$ is increased and $\varepsilon$ is held fixed, an improvement in energy conservation is observed. These parameters have been chosen to obtain an energy conservation of better than 0.1 per cent for acceptable models, see Table 2.

In agreement with Theis (1998), the Plummer softening is less collisional and has a larger relaxation time than the spline softening, even when the correspondence is made by equating their potentials depths. He shows, however, that the same relaxation time can be obtained with both the spline softening and the Plummer softening for $h \gtrsim 3 \varepsilon$. As a consequence, in order to maintain the same degree of relaxation and to resolve the vertical structure of the disc when the spline softening is used, many more particles are required than for the Plummer model. On the other hand, the Plummer softening, in comparison with the spline softening, has a stronger stabilizing effect (Romeo 1997, 1998). This may lead, as we have seen, to a late bar emergence. Further investigations of galaxy model stability using adaptive softening kernels would be worthwhile (Dehnen 2001).

This work was in part motivated to explain the difference in the characteristics of the resulting bars in various authors galaxy collision simulations. For example, works of Barnes $(1992,1998)$ show very strong and prominent bars that formed after the first encounter. On the other hand, in calculations made by Dubinski et al. (1996) the apparent bars are not observed. Although the difference may be due to different galaxy models, we decided to verify the numerical aspects of the problem. For example, both galaxy models have roughly the same number of particles and the time-step, but $\varepsilon$ used by Barnes (1998) is six times larger than Dubinski's. 
Our collision simulations of tidally triggered bar formation showed that the bar properties depend on the selected gravitational softening parameter. Within the studied ranges of $\varepsilon$ and $N$, tidal bars are shorter for smaller $\varepsilon$. Indeed, for sufficiently small $\varepsilon$ and $N$, tidal distortions can be suppressed. For example, we performed some additional collision simulations with $\varepsilon=0.0005$ and $\Delta t=1 / 1024$ for $N=40960$ and $N=163840$, where we were able to completely suppress the bar formation, and obtained small dense discs. Such models are however highly collisional and thus cannot be considered as correct. On the other hand, the bar's pattern speed and the bar's length vary weakly with $\varepsilon$ and tend to converge to a single value already for $N \gtrsim 491520$. Certainly, simulations with several million particles will approach further convergence and will show many more bar details (O'Neill \& Dubinski 2003), but an adequate $\varepsilon$ and $\Delta t$ still have to be chosen.

Confirming the results of Berentzen et al. (2004), we observe a correlation between the bar's length and the pattern speed. We found also, that in simulations with large $\varepsilon=\lambda\left(R_{\mathrm{d}}\right)$, the bars roughly conserve their length and pattern speed at least within $3 \mathrm{Gyr}$, which is time between the first and the second collisions when a clear bar is observed. However, for smaller $\varepsilon$ the bar length increases and the rotational velocity reduces.

The collision simulations have shown that a colder disc (large $\varepsilon$ ) forms an open spiral pattern, corresponding to a late type galaxy, whereas the spiral arms in a hot disc (small $\varepsilon$ ) are tightly wound. This is explained by the dispersion relation for WKB long-branch waves if the disc's perturbation is weak (Toomre 1981; Binney \& Tremaine 1987). Another possible explanation of tidal response differences may lie in the increased central density of relaxed models for small $\varepsilon$, which may create the inner Lindblad resonance that prevents the feedback of swing amplification mechanism (Norman et al. 1996).

The wiggle effect observed in our simulations could imply that some real barred galaxies do not necessarily have trailing arms. A detailed study of this effect, including gas dynamics, would be worth.

\section{Conclusions}

In this work we have investigated the influence of the triad of numerical parameters $(N, \varepsilon, \Delta t)$ on the properties of an equilibrium isolated galaxy model and the dynamics of two interacting galaxies. The main results for the galaxy equilibrium models can be summarized as follows:

1. The transfer of the disc angular momentum to the bulge and the halo depends on $\varepsilon, N$, and type of softening.

2. The convergence study indicates that for $N=491520$ and $\varepsilon=$ [0.001-0.01], the range of final values of $Q$ changes only by 14 per cent, while for smaller $N$, the $\varepsilon$ and $Q$ range broadens. Further decrease of numerical heating is observed for larger $N$ simulations, namely, for $N=1310720$, $Q$ only varies 5 per cent.

3. The final value of the Toomre stability parameter decreases linearly with the increasing of the Plummer softening.
4. For the same values of $N, \Delta t$ and $\varepsilon$, the spline softened simulations produce bars earlier than those performed with Plummer softening.

For the galaxy collision models it may be concluded that:

1. The susceptibility of galaxy models to tidal bar formation and its dissolution depend strongly on the chosen numerical parameters. Therefore, carefully chosen parameters have to be taken for specific simulations.

2. For sufficiently small $N$, the size of the bar formed during an encounter, may become too long or even not appear depending on the value of $\varepsilon$. With the increase of $N$ artificial effect of the softening is reduced.

3. The short spiral arms that are formed at the ends of the bar show a periodic change from trailing to leading and viceversa - the wiggle.

4. The spline softening gives smaller bars than in case of the Plummer softening even when they have the same potential depth.

5. The properties of the merger remnant are also affected by the softening. The remnants of cold systems maintain their barred thick disc shape, whereas those of hot systems produce rather an ellipsoid.

Acknowledgements. This work has been partially supported by the Mexican Consejo Nacional de Ciencia y Tecnología (CONACyT) under contracts U43534-R, 44917-F and J200.476/2004, and the DFG and DAAD of Germany. RFG also acknowledges the Ministry of Foreign Affairs of Mexico - "Secretaria de Relaciones Exteriores" for financial support. Some of the runs were performed at the Altix 370 computer facility kindly provided by SGI. We also thank the referee for very useful comments that improved the presentation of the paper.

\section{References}

Aarseth, S. J. 1963, MNRAS, 126, 223

Athanassoula, E. 2002, ApJ, 569, L83

Athanassoula, E. 2003, MNRAS, 341, 1179

Athanassoula, E., \& Misiriotis, A. 2002, MNRAS, 330, 35

Athanassoula, E., \& Sellwood, J. A. 1986, MNRAS, 221, 213

Athanassoula, E., Bosma, A., Lambert, J. C., \& Makino, J. 1998, MNRAS, 293, 369

Athanassoula, E., Fady, E., Lambert, J. C., \& Bosma, A. 2000, MNRAS, 314, 475

Athanassoula, E., Vozikis, Ch. L., \& Lambert, J. C. 2001, A\&A, 376, 1135

Barnes, J. E. 1992, ApJ, 393, 484

Barnes, J. E. 1998, in Galaxies: Interaction and Induced Star Formation, ed. D. Friedly, L. Martinet, \& D. Pfenniger (Berlin: Springer-Verlag), 275

Barnes, J. E., \& Hut, P. 1986, Nature, 324, 446

Berentzen, I., Athanassoula, E., Heller, C. H., \& Fricke, K. J. 2004, MNRAS, 347, 22

Binney, J., \& Tremaine, S. 1994, Galactic dynamics (Princeton NJ: Princeton Univ. Press)

Elmegreen, B. G., \& Elmegreen, D. M. 1985, ApJ, 288, 438

Debattista, V. P., \& Sellwood, J. A. 1998, ApJ, 493, L5

Debattista, V. P., \& Sellwood, J. A. 2000, ApJ, 543, 704

Dehnen, W. 1993, MNRAS, 265, 250

Dehnen, W. 2001, MNRAS, 324, 273

Dubinski, J., Mihos, J. Ch., \& Hernquist, L. 1996, ApJ, 462, 576 
Dyer, C. C., \& Ip, P. S. S. 1993, ApJ, 409, 60

Farouki, R. T., \& Salpeter, E. E. 1994, ApJ, 427, 676

Freeman, K. C. 1970, ApJ, 160, 811

Gerin, M., Combes, F., \& Athanassoula, E. 1990, A\&A, 230, 37

Goodman, J., Heggie, D. C., \& Hut, p. 1993, ApJ, 415, 715

Hayes, W. B. 2003, ApJ, 587, L59

Hernquist, L. 1987, ApJ, 64, 715

Hernquist, L. 1990, ApJ, 356, 359

Hernquist, L. 1993a, ApJS, 86, 389

Hernquist, L. 1993b, ApJ, 402, L85

Hernquist, L., \& Barnes, J. E. 1990, ApJ, 349, 562

Hernquist, L., \& Katz, N. 1989, ApJS, 70, 419

Hohl, F. 1971, ApJ, 168, 343

Huang, S., Dubinski, J., \& Carlberg, R. G. 1993, ApJ, 404, 73

Merrit, D. 1996, AJ, 111, 2462

Mihos, J. C., McGaugh, S., \& de Blok, W. J. G. 1997, ApJ, 477, L79

Miller, R. H. 1971, Ap\&SS, 14, 73

Miwa, T., \& Nogushi, M. 1998, ApJ, 499, 149

Morozov, A. G. 1981, Sov. Astron. Lett., 7, 109

Nogushi, M. 1987, MNRAS, 228, 635

Norman, C. A., Sellwood, J. A., \& Hasan, H. 1996, ApJ, 462, 114

O’Neill, J. K., \& Dubinski, J. 2003, MNRAS, 346, 251
Ostriker, J. P., \& Peebles, P. J. E. 1973, ApJ, 186, 467

Plummer, H. C. 1911, MNRAS, 71, 460

Power, C., Navarro, J. F., Jenkins, A., et al. 2003, MNRAS, 338, 14

Romeo, A. B. 1994, A\&A, 286, 799

Romeo, A. B. 1997, A\&A, 324, 523

Romeo, A. B. 1998, A\&A, 335, 922

Salo, H. 1990, A\&A, 243, 118

Sellwood, J. A. 1981, A\&A, 99, 362

Sellwood, J. A., \& Athanassoula, E. 1986, MNRAS, 221, 195

Sellwood, J. A., \& Carlberg, R. G. 1984, ApJ, 282, 61

Shibata, M., Karino, S., \& Eriguchi, Y. 2003, MNRAS, 343, 619

Springel, V., Yoshida, N., \& White, S. D. M. 2001, New Astron., 6, 79

Theis, Ch. 1998, A\&A, 330, 1180

Toomre, A. 1964, ApJ, 139, 1217

Toomre, A. 1981, in The Structure and Evolution of Normal Galaxies, ed. M. Fall, \& D. Lynden-Bell (Cambridge: Cambridge Univ. Press), 111

Valenzuela, O., \& Klypin, A. 2003, MNRAS, 345, 406

Walker, I. R., Mihos, J. Ch., \& Hernquist, L. 1996, ApJ, 460, 121

Weinberg, M. D. 1985, MNRAS, 213, 451

White, R. L. 1988, ApJ, 330, 26

Zotov, V. M., \& Morozov, A. G. 1987, Soviet Astron. Lett., 13, 136 